UdeM-GPP-TH-05-132

$\mathrm{IPPP} / 05 / 10$

$\mathrm{DCPT} / 05 / 20$

KIAS-P05030

KAIST-TH 2005/07

FTUAM 05/06

IFT-UAM/CSIC-05-21

hep-ph/0505019

May 2005

\title{
Direct detection of neutralino dark matter in supergravity
}

\author{
S. Baek ${ }^{1}$, D.G. Cerdeño ${ }^{2}$ Y.G. Kim ${ }^{3}$, P. Ko ${ }^{4}$, and C. Muñoz ${ }^{5,6}$ \\ ${ }^{1}$ Laboratoire René J.-A. Lévesque, Université de Montréal, C.P. 6128, succ. centre-ville, \\ Montréal, QC, Canada HЗC 3J7 \\ ${ }^{2}$ Institute for Particle Physics Phenomenology, University of Durham, DH1 3LE, UK \\ 3 Department of Physics, Korea University, Seoul 136-701, Korea \\ ${ }^{4}$ School of Physics, KIAS, Seoul 130-722, Korea \\ ${ }^{5}$ Department of Physics, KAIST, Daejon 305-701, Korea \\ ${ }^{6}$ Departamento de Física Teórica $C$-XI and Instituto de Física Teórica C-XVI, \\ Universidad Autónoma de Madrid, Cantoblanco, 28049 Madrid, Spain.
}

\begin{abstract}
The direct detection of neutralino dark matter is analysed in general supergravity scenarios, where non-universal soft scalar and gaugino masses can be present. In particular, the theoretical predictions for the neutralino-nucleon cross section are studied and compared with the sensitivity of dark matter detectors. We take into account the most recent astrophysical and experimental constraints on the parameter space, including the current limit on $\mathrm{B}\left(B_{s}^{0} \rightarrow \mu^{+} \mu^{-}\right)$. The latter puts severe limitations on the dark matter scattering cross section, ruling out most of the regions that would be within the reach of present experiments. We show how this constraint can be softened with the help of appropriate choices of non-universal parameters which increase the Higgsino composition of the lightest neutralino and minimise the chargino contribution to the $b \rightarrow s$ transition.
\end{abstract}




\section{Introduction}

A long-lived or stable weakly interacting massive particle (WIMP) is a particularly attractive candidate for dark matter in the Universe [1]. The interest in WIMPs resides mainly on the fact that they can be present in the right amount to account for the matter density observed in the analysis of galactic rotation curves [2], cluster of galaxies and large scale flows [3], $0.1 \lesssim \Omega h^{2} \lesssim 0.3\left(0.094 \lesssim \Omega h^{2} \lesssim 0.129\right.$ if we take into account the recent data obtained by the WMAP satellite [4]).

Many underground experiments are being carried out around the world in order to detect the flux of WIMPs on the Earth, by observing their elastic scattering on target nuclei through nuclear recoils [1]. Although one of the current experiments, the DAMA collaboration [5], has reported data favouring the existence of a WIMP signal with a WIMP-proton cross section $\approx 10^{-6}-10^{-5} \mathrm{pb}$ for a WIMP mass smaller than 500-900 GeV [5, 6], other collaborations such as CDMS Soudan [7], EDELWEISS [8], and ZEPLIN I [9] claim to have excluded important regions of the DAMA parameter space. In the light of these experimental results more than 20 experiments are running or in preparations around the world. For example, this is the case of GEDEON [10], which will be able to explore positively a WIMP-nucleon cross section $\sigma \gtrsim 3 \times 10^{-8}$ pb. CDMS Soudan will be able to test in the future $\sigma \approx 2 \times 10^{-8} \mathrm{pb}$, and the very sensitive detector GENIUS [11] will test a WIMP-nucleon cross section $\sigma \approx 10^{-9} \mathrm{pb}$. In fact, already planned detectors working with 1 tonne of Ge/Xe [12] are expected to reach cross sections as low as $10^{-10} \mathrm{pb}$.

The leading candidate for WIMP is the lightest neutralino, $\tilde{\chi}_{1}^{0}$, a particle predicted by the supersymmetric (SUSY) extension of the standard model. Given the experimental situation, and assuming that the dark matter is a neutralino, it is natural to wonder how big the cross section for its direct detection can be. Obviously, this analysis is crucial in order to know the possibility of detecting dark matter in the experiments. In fact, the analysis of the neutralino-proton cross section has been carried out by many authors and during many years [1]. The most recent studies take into account the present experimental and astrophysical constraints on the parameter space. Concerning the former, the lower bound on the Higgs mass, the $b \rightarrow s \gamma$ branching ratio, and the muon anomalous magnetic moment, $a_{\mu} \equiv\left(g_{\mu}-2\right) / 2$, have been considered. The astrophysical bounds on the dark matter density, have also been imposed on the theoretical computation of the relic neutralino density, assuming thermal production. In addition, the constraints that the absence of dangerous charge and colour breaking minima imposes on the parameter space have also been taken into account [13].

Recently, the importance of the improved experimental upper limit on the $B_{s}^{0} \rightarrow$ $\mu^{+} \mu^{-}$branching ratio [14, 15] was stressed in Ref. [16], where a strong correlation was found between this observable and the spin-independent neutralino-nucleon cross section, the origin of which resides in $\tan \beta$ and the neutral Higgs boson masses $\left(m_{H}, m_{A}\right)$. In particular, both observables increase for large $\tan \beta$ and low values of the Higgs 
masses. For this reason, some of regions where $\sigma_{\tilde{\chi}_{1}^{0}-p}$ can be consistent with the future dark matter detectors are excluded.

We will work within the context of supergravity theories, taking the soft supersymmetry-breaking parameters of the MSSM as inputs at the Grand Unification scale, $M_{G U T} \approx 2 \times 10^{16} \mathrm{GeV}$, and solving the renormalization group equations (RGEs) to obtain the supersymmetric spectrum at the electroweak scale. In the particular case of the minimal supergravity scenario (mSUGRA), where the soft terms are considered to be universal at the GUT scale, the predictions for $\mathrm{B}\left(B_{s}^{0} \rightarrow \mu^{+} \mu^{-}\right)$are currently below the reach of Tevatron [17, 16, 18]. Therefore no further constraint on the theoretical predictions for the neutralino-nucleon cross section appears and the usual upper limit, $\sigma_{\tilde{\chi}_{1}^{0}-p} \lesssim 10^{-8} \mathrm{pb}$, is obtained.

Relaxing the universality condition is a more generic situation within the framework of supergravity. The presence of non-universal soft scalar [19, 20, 21, 22, 23, 24, 25. 26, 27, 28, 29, 30, 31, 32, 13, 33, and gaugino masses [34, 27, 29, 35, 30, 36, 37, 13, has been extensively considered in the literature. Non-universalities in both the scalar and gaugino sectors have also been studied [38, 39]. Certain choices of non-universalities in these scenarios were shown to lead to a sizable increase of the theoretical predictions for $\sigma_{\tilde{\chi}_{1}^{0}-p}$. Nevertheless, in such cases the constraint on $\mathrm{B}\left(B_{s}^{0} \rightarrow \mu^{+} \mu^{-}\right)$plays a very important role and large regions in the parameter space are forbidden [16] (see also [31] for an analysis in a model with minimal $S O_{10}$ SUSY breaking).

For example, non-universal scalar masses can lead to an enhancement in $\sigma_{\tilde{\chi}_{1}^{0}-p}$, mainly due to the associated reduction of the Higgs masses. The most important effect is due to non-universal soft Higgs masses, which can be parameterised at the GUT scale as follows:

$$
m_{H_{d}}^{2}=m^{2}\left(1+\delta_{1}\right), \quad m_{H_{u}}^{2}=m^{2}\left(1+\delta_{2}\right) .
$$

The optimal choice to increase the neutralino detection cross section is $\delta_{1}<0$ and $\delta_{2}>0$. Nevertheless, the decrease in the Higgs masses also leads to large values of $\mathrm{B}\left(B_{s}^{0} \rightarrow \mu^{+} \mu^{-}\right)$, which can be in conflict with the experimental constraint. As a consequence, those regions of the parameter space where the neutralino scattering is within the reach of the DAMA experiment are ruled out. Actually, in some cases, the upper bound on the $\mathrm{B}\left(B_{s}^{0} \rightarrow \mu^{+} \mu^{-}\right)$branching ratio becomes even stronger than the upper bound from CDMS experiment [16].

On the other hand, non-universal gaugino masses allow more flexibility in the neutralino sector. The following parameterisation can be used

$$
M_{1}=M, \quad M_{2}=M\left(1+\delta_{2}^{\prime}\right), \quad M_{3}=M\left(1+\delta_{3}^{\prime}\right),
$$

where $M_{1,2,3}$ are the bino, wino and gluino masses, respectively, and $\delta_{i}^{\prime}=0$ corresponds to the universal case. A decrease in $M_{3}$ can induce both a decrease in the Higgs mass, through its effect on the RGE of $m_{H_{u}}^{2}$, and an increase of the Higgsino components of $\tilde{\chi}_{1}^{0}$. Despite the associated enhancement of $\sigma_{\tilde{\chi}_{1}^{0}-p}$, the current limit on $\mathrm{B}\left(B_{s}^{0} \rightarrow \mu^{+} \mu^{-}\right)$ puts a strong constraint in the large $\tan \beta$ region. 
In this paper we analyse the most general situation, where both scalar and gaugino masses are allowed to be non-universal at $M_{G U T}$. This is an interesting possibility, since neutralinos whose detection cross section can be within the reach of future experiments can appear with a wide range of masses, from over $400 \mathrm{GeV}$ to almost $10 \mathrm{GeV}$ [39. As we will see, the new experimental result for $\mathrm{B}\left(B_{s}^{0} \rightarrow \mu^{+} \mu^{-}\right)$has an important effect.

\section{Non-universal scalar and gaugino masses}

In our analysis the soft supersymmetry-breaking terms, parameterised according to (11) and (2), are taken as inputs at the high energy scale $M_{G U T}$, where unification of the gauge coupling constants takes place. In addition, the ratio of the Higgs vacuum expectation values, $\tan \beta \equiv\left\langle H_{u}^{0}\right\rangle /\left\langle H_{d}^{0}\right\rangle$ is a free parameter, as well as the sign of the Higgsino mass parameter, $\mu$, which remains undetermined by the minimisation of the Higgs potential.

The most recent experimental and astrophysical constraints will be taken into account. In particular, the lower bounds on the masses of the supersymmetric particles and on the lightest Higgs have been implemented, as well as the experimental bounds on the branching ratio of the $b \rightarrow s \gamma$ process $\left(2 \times 10^{-4} \leq \mathrm{B}(b \rightarrow s \gamma)\right) \leq 4.1 \times 10^{-4}$ from the measurements of $B \rightarrow X_{s} \gamma$ decays at CLEO [40] and BELLE [4]) and on $a_{\mu}^{\mathrm{SUSY}}$. The evaluation of the neutralino relic density is carried out with the program micrOMEGAs [42], and, due to its relevance, the effect of the WMAP constraint on it will be shown explicitly. Dangerous charge and colour breaking minima of the Higgs potential will be avoided by excluding unbounded from below (UFB) directions. Finally, the improved experimental constraint on the $B_{s}^{0} \rightarrow \mu^{+} \mu^{-}$branching ratio, $\mathrm{B}\left(B_{s}^{0} \rightarrow \mu^{+} \mu^{-}\right)<2.9 \times 10^{-7}$, obtained from a combination of the results of CDF [14] and D0, [15], will be included.

Concerning $a_{\mu}^{\mathrm{SUSY}}$, we have taken into account the recent experimental result for the muon anomalous magnetic moment [43, as well as the most recent theoretical evaluations of the Standard Model contributions [44, 45, 46]. It is found that when $e^{+} e^{-}$ data are used the experimental excess in $a_{\mu}$ would constrain a possible supersymmetric contribution to be $a_{\mu}^{\text {SUSY }}=(27.1 \pm 10) \times 10^{-10}$ 44]. However, when tau data are used a smaller discrepancy with the above experimental measurement is found. Due to this reason, in our analysis we will not impose this constraint, but only indicate the regions compatible with it at $2 \sigma$ level, this is, $7.1 \times 10^{-10} \leq a_{\mu}^{\mathrm{SUSY}} \leq 47.1 \times 10^{-10}$.

Even after including the tau data, positive values of $a_{\mu}^{\text {SUSY }}$ are clearly favoured. Because of this and the fact that the sign of $a_{\mu}^{\mathrm{SUSY}}$ is basically given by $\mu M_{2}$, we will only consider the case where $\operatorname{sign}\left(M_{2}\right)=\operatorname{sign}(\mu)^{1}$. Similarly, the constraint on the

\footnotetext{
${ }^{1} \mathrm{~A}$ different sign for $M_{2}$ and $\mu$ could in principle also be used, thus obtaining $a_{\mu}^{\mathrm{SUSY}}<0$. Nevertheless, since the negative values of $a_{\mu}^{\mathrm{SUSY}}$ which are allowed by tau data are small in modulus, very
} 
$b \rightarrow s \gamma$ branching ratio is much weaker when $\operatorname{sign}\left(M_{3}\right)=\operatorname{sign}(\mu)$. Finally, variations in the sign of $M_{1}$ do not induce significant changes in the allowed regions of the parameter space (e.g., its effect on $a_{\mu}^{\mathrm{SUSY}}$, due to diagrams with neutralino intermediate states, is smaller than the one of $\left.M_{2}\right)$. However, when $\operatorname{sign}\left(M_{1}\right)=\operatorname{sign}(\mu)$ the theoretical predictions for $\sigma_{\tilde{\chi}_{1}^{0}-p}$ are larger. For these reasons we will restrict our analysis to positive values of $M_{1,2,3}$ and $\mu>0$. Note in this sense, that due to the symmetry of the RGEs, the results for $\left(M_{1,2,3}, \mu, A\right)$ are identical to those for $\left(-M_{1,2,3},-\mu,-A\right)$.

We will be mostly interested in analysing the conditions under which high values for the cross section are obtained. For this reason, we will concentrate on some interesting choices for scalar non-universalities, exemplified by the following cases [13]

$$
\begin{array}{ll}
\text { a) } \quad \delta_{1}=0, & \delta_{2}=1 ; \\
\text { b) } \quad \delta_{1}=-1, & \delta_{2}=0 ; \\
\text { c) } \quad \delta_{1}=-1, & \delta_{2}=1,
\end{array}
$$

and study the effect of adding gaugino non-universalities to these.

For illustrative purposes, let us first briefly review the case with only non-universal Higgs masses.

For low values of $\tan \beta(\tan \beta \lesssim 20)$ the most important constraint on the parameter space is that on the lightest Higgs mass, which excludes regions with low values for the common gaugino mass, $M$. This sets an upper bound for the neutralino-nucleon cross section such that compatibility with the present dark matter experiments is not achieved. In these cases the region excluded due to the constraint on $\mathrm{B}\left(B_{s}^{0} \rightarrow \mu^{+} \mu^{-}\right)$ is small and contained within the area excluded by the Higgs constraint. Therefore, it does not lead to a further restriction in the allowed area. It is worth reminding that thanks to the Higgs non-universality the UFB constraints are more easily fulfilled than in mSUGRA [13].

The theoretical predictions for the neutralino-nucleon cross section increase for larger values of $\tan \beta$. However, at the same time the experimental constraints on $b \rightarrow s \gamma$ and $B_{s}^{0} \rightarrow \mu^{+} \mu^{-}$become more stringent ${ }^{2}$ and can exclude those regions with larger values of $\sigma_{\tilde{\chi}_{1}^{0}-p}$ [16].

Let us illustrate this with an example. The theoretical predictions for the neutralinonucleon cross section as a function of the neutralino mass are represented in Fig. 11 for a scan in the parameter space where $m \leq 1500 \mathrm{GeV}$ and $50 \mathrm{GeV} \leq M \leq 1500 \mathrm{GeV}$, with $\tan \beta=35, A=0$, and the three choices of non-universal Higgs masses (3). Light grey dots in the figure represent points fulfilling all the experimental and UFB constraints. Dark grey dots correspond to those points where the neutralino relic density falls in

large values of $\left|M_{2}\right|$ are necessary. Therefore this possibility is very constrained.

${ }^{2}$ Notice, however, that when a general flavour mixing among squarks is taken into account, a calculation of $\mathrm{B}\left(B_{s}^{0} \rightarrow \mu^{+} \mu^{-}\right)$beyond the leading order in the large $\tan \beta$ regime may show a reduction with respect to its value under the assumption of minimal flavour violation [4]. 


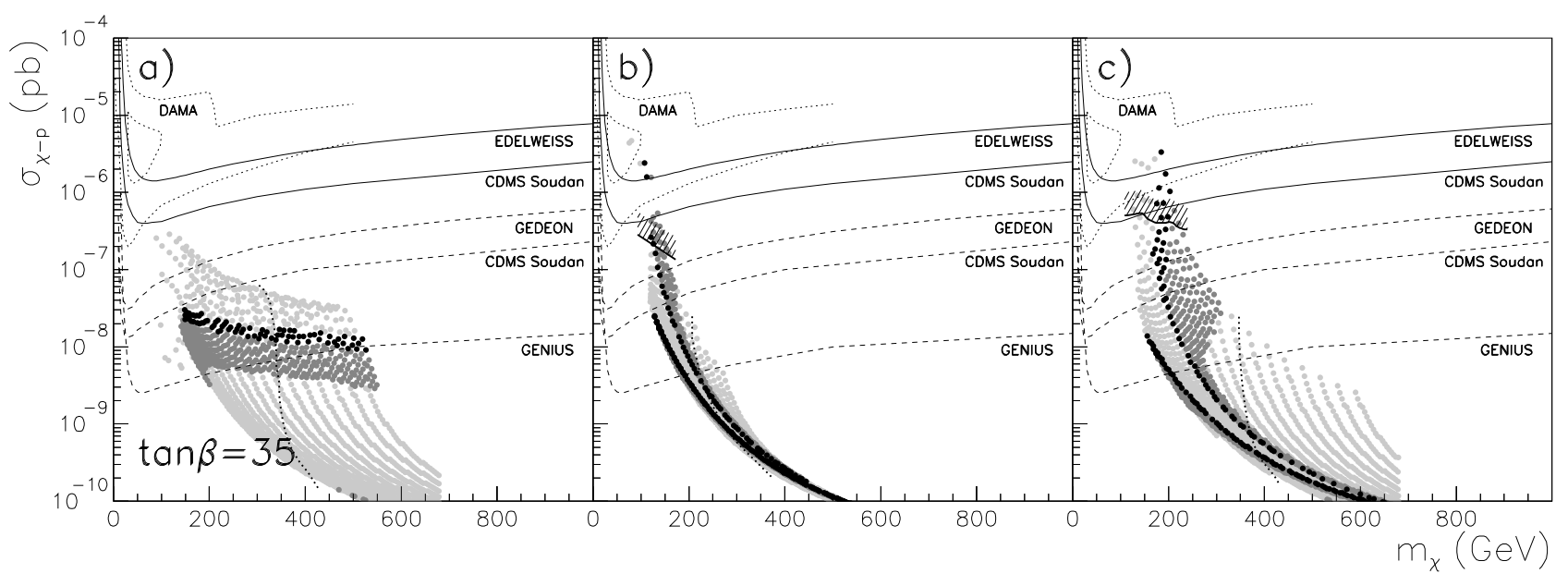

Figure 1: Scatter plot of the scalar neutralino-proton cross section $\sigma_{\tilde{\chi}_{1}^{0}-p}$ as a function of the neutralino mass $m_{\tilde{\chi}_{1}^{0}}$ for universal gauginos, $\delta_{2,3}^{\prime}=0$, and the three choices for nonuniversal scalars (3) in a case with $\tan \beta=35$ and $A=0$. The light grey dots correspond to points fulfilling the experimental and UFB constraints. The dark grey dots represent points fulfilling in addition $0.1 \leq \Omega_{\tilde{\chi}_{1}^{0}} h^{2} \leq 0.3$ and the black ones correspond to those consistent with the WMAP range. The area to the right of the dotted line is disfavoured by $g_{\mu}-2$ if $e^{+} e^{-}$data is used. Points above the solid line with upper shading are excluded by the experimental constraint on $\mathrm{B}\left(B_{s}^{0} \rightarrow \mu^{+} \mu^{-}\right)$. The sensitivities of present and projected dark matter experiments are also depicted with solid and dashed lines, respectively. The large (small) area bounded by dotted lines is allowed by the DAMA experiment when astrophysical uncertainties are (are not) taken into account.

the range $0.1 \leq \Omega_{\tilde{\chi}_{1}^{0}} h^{2} \leq 0.3$, and those points consistent with the WMAP range are represented in black. The region to the right of the dotted line does not fulfil the constraint on $a_{\mu}^{\text {SUSY }}$ when $e^{+} e^{-}$data are used. Due to the importance of the constraint on $\mathrm{B}\left(B_{s}^{0} \rightarrow \mu^{+} \mu^{-}\right)$, its effect is shown explicitly in the figure by means of a solid line with upper shading. Points above that line are excluded for having a too large value of $\mathrm{B}\left(B_{s}^{0} \rightarrow \mu^{+} \mu^{-}\right)$.

There are obvious differences in the predicted $\sigma_{\tilde{\chi}_{1}^{0}-p}$ obtained in case a) and in cases b) and c). Whereas in case a) the non-universal structure of the Higgs parameters is such that the $\mu$ term is particularly reduced, in cases b) and c) it is the decrease in $m_{A}$ which is more important, owing to the decrease of $m_{H_{d}}^{2}$ at the GUT scale. This entails a larger increase of $\sigma_{\tilde{\chi}_{1}^{0}-p}$ in these last cases, but also leads to an enhanced $\mathrm{B}\left(B_{s}^{0} \rightarrow \mu^{+} \mu^{-}\right)$ which can be in conflict with the experimental bound. As a consequence of this, all the points with $\sigma_{\tilde{\chi}_{1}^{0}-p} \gtrsim 10^{-6} \mathrm{pb}$ are ruled out. Part of the remaining points are within the reach of the projected GEDEON detector. In case a) $\sigma_{\tilde{\chi}_{1}^{0}-p} \lesssim 3 \times 10^{-8} \mathrm{pb}$ and would escape detection in the projected CDMS Soudan experiment and could only be tested in the GENIUS detector. 


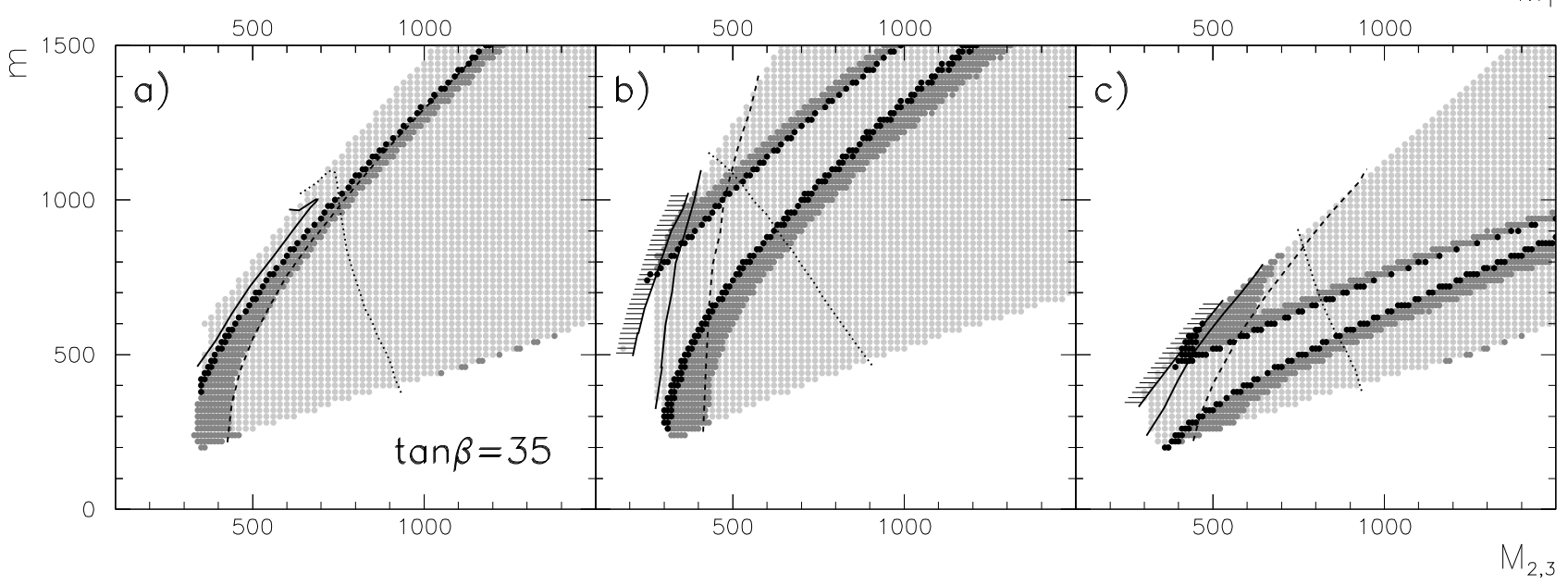

Figure 2: Effect of the different experimental constraints on the parameter space $\left(m, M_{i}\right)$ for universal gauginos, $\delta_{2,3}^{\prime}=0$, and the three choices for non-universal scalars (3) in a case with $\tan \beta=35$ and $A=0$. The region to the right of the dotted line is disfavoured by $g_{\mu}-2$ if $e^{+} e^{-}$data is used. The area to the left of the solid line with upper shading is ruled out by the experimental constraint on $\mathrm{B}\left(B_{s}^{0} \rightarrow \mu^{+} \mu^{-}\right)$. The light shaded area is favoured by the remaining experimental and UFB constraints, while the dark one fulfils in addition $0.1 \leq \Omega_{\tilde{\chi}_{1}^{0}} h^{2} \leq 0.3$. The black region on top of this indicates the WMAP range, $0.094 \leq \Omega_{\tilde{\chi}_{1}^{0}} h^{2} \leq 0.129$. The zone to the left of the thick solid line is within the projected sensitivity of the CDMS Soudan experiment. Similarly, the region to the left of the thick dashed line corresponds to those points that will be accessible to the GENIUS experiment.

To understand the effect of the different constraints it is illustrative to represent the excluded regions in the $(m, M)$ parameter space. This is done in Fig.2, where the same colour convention as in Fig. [1 is used for denoting points with different values of the relic density. Points to the left of the solid line with upper shading are excluded by the bound on $\mathrm{B}\left(B_{s}^{0} \rightarrow \mu^{+} \mu^{-}\right)$, and those to the upper right of the dotted line are disfavoured by $g_{\mu}-2$ when $e^{+} e^{-}$data are used. The regions compatible with the sensitivities of the projected CDMS Soudan and GENIUS detectors correspond to those on the left of the solid and dashed lines, respectively.

In case a) all the points within the reach of the projected CDMS Soudan detector lie in the region where $\Omega_{\tilde{\chi}_{1}^{0}} h^{2}<0.094$, in the upper left corner. However, most of the points in agreement with the WMAP result (black dots) are within the reach of GENIUS. Interestingly, this occurs for a wide range of values of $m$ and $M$. Contrariwise, in cases b) and c) CDMS Soudan can test some of the points in agreement with WMAP. This occurs when the CP-odd Higgs mass is sufficiently small, in the proximity of the region which is ruled out for having $m_{A}^{2}<0$, in the upper left corner of the plot. Notice that this is the case for $M \lesssim 250 \mathrm{GeV}$, and a part of those points is disallowed by the constraint on $\mathrm{B}\left(B_{s}^{0} \rightarrow \mu^{+} \mu^{-}\right)$. In these two cases, points with $M \lesssim 550 \mathrm{GeV}$ could be 


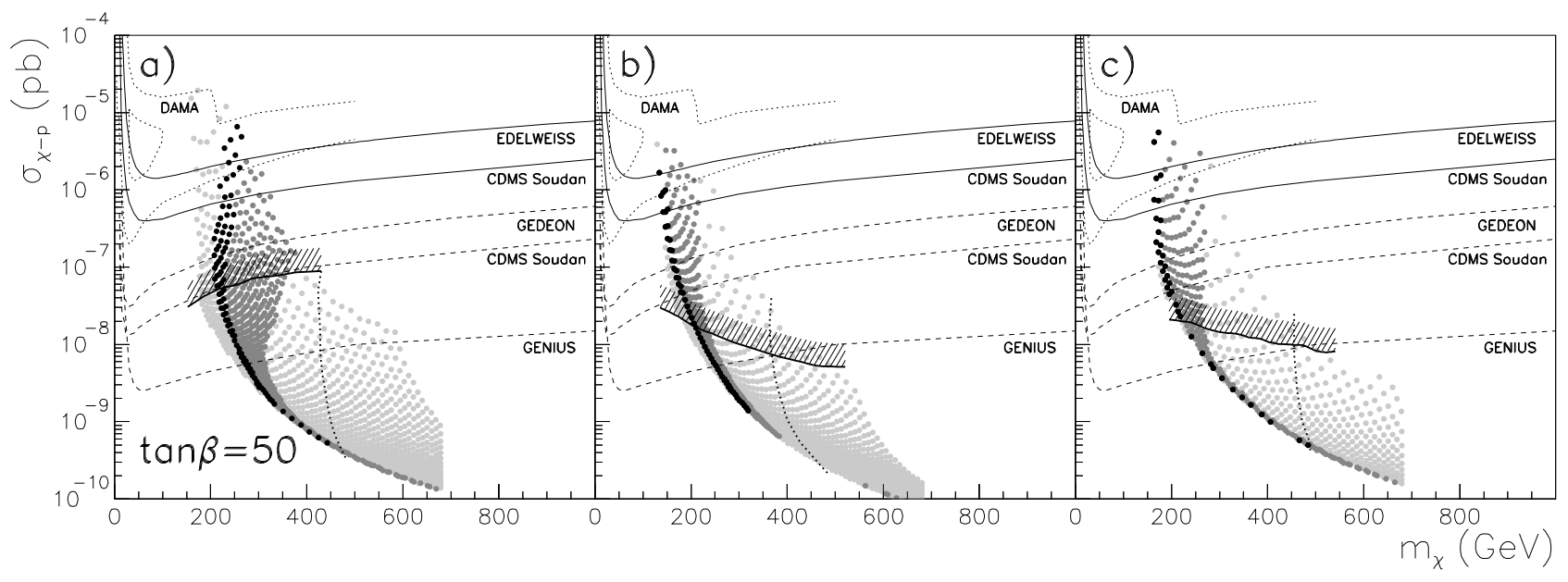

Figure 3: The same as Fig.1, but for $\tan \beta=50$.

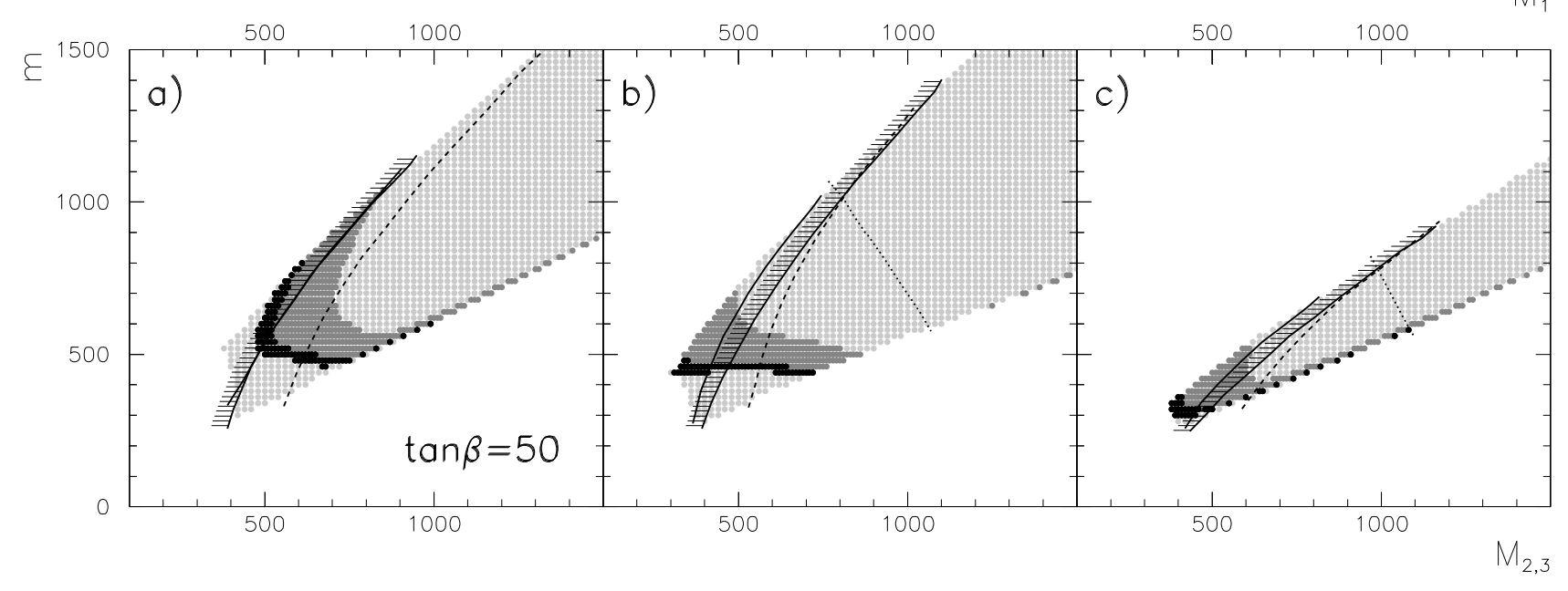

Figure 4: The same as Fig.2, but for $\tan \beta=50$.

tested by GENIUS.

With larger values of $\tan \beta$ the resulting $\sigma_{\tilde{\chi}_{1}^{0}-p}$ could be even within the reach of present dark matter detectors. Let us study this possibility and concentrate on the case $\tan \beta=50$. The corresponding theoretical predictions for $\sigma_{\tilde{\chi}_{1}^{0}-p}$ are represented in Fig. [3. Although in principle, regions of the parameter space are found where the neutralino detection cross section can be as large as $\sigma_{\tilde{\chi}_{1}^{0}-p} \gtrsim 10^{-6} \mathrm{pb}$ while having the correct relic density, the associated increase in $\mathrm{B}\left(B_{s}^{0} \rightarrow \mu^{+} \mu^{-}\right)$is such that the experimental constraint is violated and extensive areas are ruled out. Owing to this, points compatible with present detector sensitivities disappear and an upper bound of $\sigma_{\tilde{\chi}_{1}^{0}-p} \lesssim 10^{-7}$ pb is obtained. 
The corresponding $(m, M)$ parameter space is plotted in Fig.4. For such high values of $\tan \beta$ the three choices of non-universal Higgs masses, a), b), and c) allow a large reduction in the CP-odd Higgs mass. In particular, this reduction is larger than the decrease of $\mu$ in case a) and for this reason the three examples present a large resemblance. Once more the regions which are within the reach of dark matter detectors are very disfavoured by the predicted values of $\mathrm{B}\left(B_{s}^{0} \rightarrow \mu^{+} \mu^{-}\right)$. As we can see, in the three cases the areas excluded by this constraint enclose all the points within the reach of the projected CDMS Soudan. GENIUS would be able to test some of the remaining points which have the correct value for the relic density. Note that most of the points of the parameter space which could escape detection at GENIUS are located along the coannihilation tail, where the neutralino and the light stau are almost degenerate in mass.

At this point it may seem that the observed correlation between $\mathrm{B}\left(B_{s}^{0} \rightarrow \mu^{+} \mu^{-}\right)$and $\sigma_{\tilde{\chi}_{1}^{0}-p}$ is inevitable and that therefore large neutralino detection cross sections, within the reach of present experiments, are not attainable. However, this correlation can be diluted under several circumstances. For instance, the gluino mediated contribution to the $b \rightarrow s$ transition can have the opposite sign than the chargino mediated term, which is typically dominant, thereby leading to a partial cancellation and slightly decreasing $\mathrm{B}\left(B_{s}^{0} \rightarrow \mu^{+} \mu^{-}\right)$.

For a larger reduction, one can consider tuning the value of the top trilinear coupling, $A_{t}$, at the GUT scale in such a way that the stop $\left(\tilde{t}_{L}-\tilde{t}_{R}\right)$ mixing is reduced and the stop mass increased. Consequently, the chargino mediated $b \rightarrow s$ transition is suppressed. This can be done with $A_{t}>0$ at the GUT scale. For large values of $\tan \beta$, for which the $\mu / \tan \beta$ term in the stop mixing can be neglected, the chargino contribution to $\mathrm{B}\left(B_{s}^{0} \rightarrow \mu^{+} \mu^{-}\right)$can be qualitatively expressed as

$$
\mathrm{B}\left(B_{s}^{0} \rightarrow \mu^{+} \mu^{-}\right) \propto \frac{\tan ^{6} \beta}{m_{A}^{4}}\left(\frac{\mu A_{t}}{m_{\tilde{t}_{L}}^{2}}\right)^{2} .
$$

When larger and positive values for $A_{t}$ are taken at the GUT scale, its value at the electroweak scale, after applying the RGEs, becomes less negative. Thus $A_{t}^{2}$ decreases, $m_{\tilde{t}_{L, R}}^{2}$ increase through the effect of $A_{t}$ on their RGEs, and as a consequence, the term in parenthesis in (41) becomes smaller. Such a modification of $A_{t}$ also causes a decrease in the lightest Higgs mass. This, together with the enhancement of the Higgsino components of $\tilde{\chi}_{1}^{0}$, is helpful for obtaining an increase in $\sigma_{\tilde{\chi}_{1}^{0}-p}$ but one has to make sure the experimental bound on $m_{h}$ is not violated.

In order to exemplify this behaviour let us concentrate on the case with $\tan \beta=50$ and non-universal Higgs masses according to case a) in (3), and consider variations in the trilinear parameter. For example, let us compare the case where $A=0$, which was already shown in Fig. 11, with the one where $A=1.4 M$. The ratio $\left(\mu A_{t} / m_{\tilde{t}_{L}}^{2}\right)^{2}$ is represented on the left hand side of Fig.5 as a function of the CP-odd Higgs mass for both cases, where we have scanned in the whole $(m, M)$ parameter space, and 

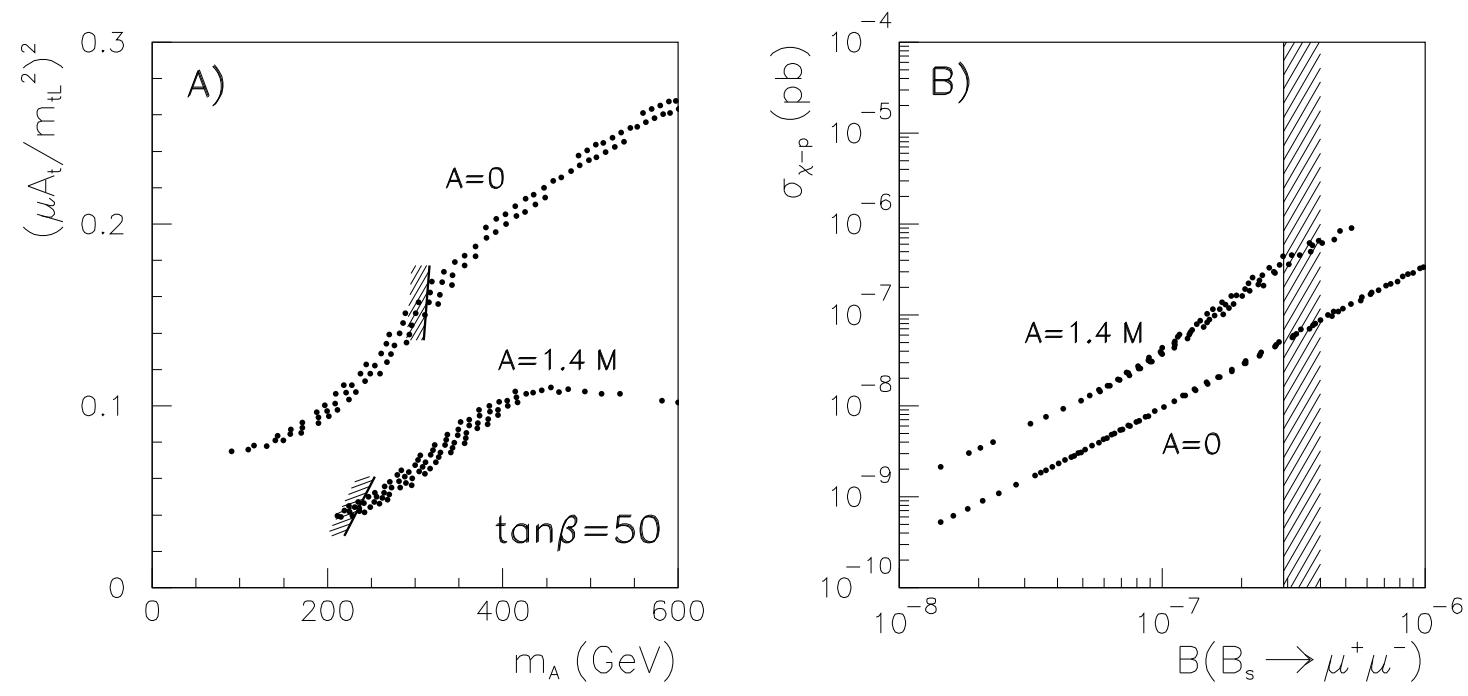

Figure 5: A) Ratio $\left(\mu A_{t} / m_{\tilde{t}_{L}}^{2}\right)^{2}$ versus the CP-odd Higgs mass for two cases with $\tan \beta=$ 50, non-universal Higgses with $\delta_{1}=0$ and $\delta_{2}=1$ in (1), and $A=0,1.4 M$. Only those points fulfilling all the experimental constraints and whose relic density is in agreement with WMAP are plotted. The effect of the upper limit on $\mathrm{B}\left(B_{s}^{0} \rightarrow \mu^{+} \mu^{-}\right)$is shown explicitly by means of a solid line with shading, which excludes the points to its left. B) Associated theoretical predictions for $\sigma_{\tilde{\chi}_{1}^{0}-p}$ versus $\mathrm{B}\left(B_{s}^{0} \rightarrow \mu^{+} \mu^{-}\right)$. The experimental limit on $\mathrm{B}\left(B_{s}^{0} \rightarrow \mu^{+} \mu^{-}\right)$is represented by the vertical line with right shading.

applied all the experimental and astrophysical constraints. As we can see, $\left(\mu A_{t} / m_{\tilde{t}_{L}}^{2}\right)^{2}$ is considerably smaller for the example with $A=1.4 M$ and for this reason the predictions for $\mathrm{B}\left(B_{s}^{0} \rightarrow \mu^{+} \mu^{-}\right)$decrease. As a consequence, smaller values of the CP-odd Higgs mass are viable. Owing to this and to the enhancement of the Higgsino composition of the lightest neutralino that originates from the decrease of $\mu$, the theoretical predictions for $\sigma_{\tilde{\chi}_{1}^{0}-p}$ become larger. This is illustrated on the right-hand side of Fig. 5, where $\sigma_{\tilde{\chi}_{1}^{0}-p}$ is represented versus $\mathrm{B}\left(B_{s}^{0} \rightarrow \mu^{+} \mu^{-}\right)$for both $A=0$ and $A=1.4 M$. An increase of one order of magnitude in $\sigma_{\tilde{\chi}_{1}^{0}-p}$ is observed for $A=1.4 M$ and the resulting detection cross section, $\sigma_{\tilde{\chi}_{1}^{0}-p} \lesssim 4 \times 10^{-7} \mathrm{pb}$ is close to the present CDMS Soudan sensitivity. Finally, the resulting neutralino detection cross section as a function of the neutralino mass is represented in Fig.6, which can be compared with Fig. [3. and evidences the less strict bound on $\mathrm{B}\left(B_{s}^{0} \rightarrow \mu^{+} \mu^{-}\right)$. For $A \gtrsim 1.4 M$ the ratio $\left(\mu A_{t} / m_{\tilde{t}_{L}}^{2}\right)^{2}$ does not decrease further and consequently the maximum value of $\sigma_{\tilde{\chi}_{1}^{0}-p}$ does not continue increasing. Needless to say, the optimal choice of $A$ that leads to maximal $\sigma_{\tilde{\chi}_{1}^{0}-p}$ is very dependent on the rest of the initial parameters.

The decrease in the ratio $\left(\mu A_{t} / m_{\tilde{t}_{L}}^{2}\right)^{2}$, and therefore in the chargino mediated $b \rightarrow s$ transition is obviously more effective in those cases where the Higgsino mixing, $\mu$, is reduced. As we have already explained, this can be done with non-universal Higgs masses where $\delta_{2}>0$ in (II). This is an interesting possibility, since a decrease of $\mu$ also 


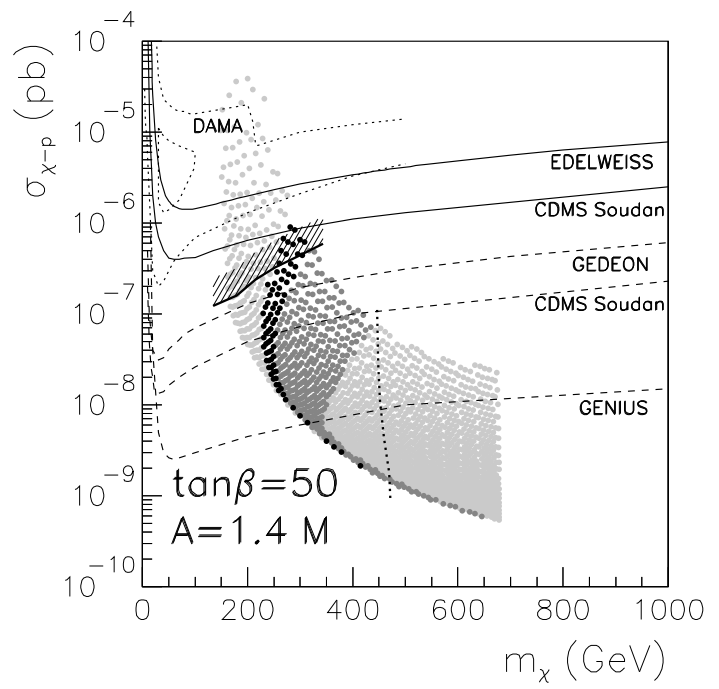

Figure 6: The same as Fig.1] but for a case with $\tan \beta=50$, non-universal Higgses with $\delta_{1}=0$ and $\delta_{2}=1$ in (1), and $A=1.4 M$.

entails an increase of the Higgsino components of the lightest neutralino and thus a larger detection cross section. Although this would favour both cases a) and c) in (31), we have already seen in Fig. [3 how the $\mathrm{B}\left(B_{s}^{0} \rightarrow \mu^{+} \mu^{-}\right)$is more stringent in the latter. This is due to the fact that in case c) the mass of the CP-odd Higgs is also reduced. Thus for the same value of $\mu, m_{A}$ is smaller and consequently $\mathrm{B}\left(B_{s}^{0} \rightarrow \mu^{+} \mu^{-}\right)$larger. In other words, case a) allows lower values for the $\mu$ parameter and lighter CP-odd Higgses. Once more, this leads to larger $\sigma_{\tilde{\chi}_{1}^{0}-p}$.

This is illustrated in Fig.7. On the left-hand side we have plotted the value of the $\mu$ term versus the CP-odd mass for cases a) and c), with $\tan \beta=50$ and $A=0$. Only those points fulfilling all the experimental constraints and having the correct relic density are displayed. Points below the solid line with left dashing correspond to those excluded by the experimental constraint on $\mathrm{B}\left(B_{s}^{0} \rightarrow \mu^{+} \mu^{-}\right)$. Whereas in case c) the CP-odd mass and $\mu$ parameter are bounded to be $m_{A} \gtrsim 350 \mathrm{GeV}$ and $\mu \gtrsim 600$ $\mathrm{GeV}$, in case a) the value of $\mathrm{B}\left(B_{s}^{0} \rightarrow \mu^{+} \mu^{-}\right)$remains below the experimental constraint for $m_{A} \gtrsim 310 \mathrm{GeV}$ and $\mu \gtrsim 475 \mathrm{GeV}$. Consequently, $\sigma_{\tilde{\chi}_{1}^{0}-p}$ is slightly higher. The corresponding predictions for $\sigma_{\tilde{\chi}_{1}^{0}-p}$ as a function of $\mathrm{B}\left(B_{s}^{0} \rightarrow \mu^{+} \mu^{-}\right)$are represented on the right-hand side. Larger values of $\delta_{2}$ can help in obtaining even larger values for $\sigma_{\tilde{\chi}_{1}^{0}-p}$ but obviously the decrease in $\mu$ is restricted by the experimental bound on the chargino mass.

With this caveat in mind, let us now discuss the effect of adding non-universal gaugino masses, which are known to provide a larger flexibility in the neutralino sector [39]. Due to the importance of the gluino mass parameter, we will group the possible gaugino non-universalities in two different cases, depending on whether the ratio $M_{3} / M_{1}$ 

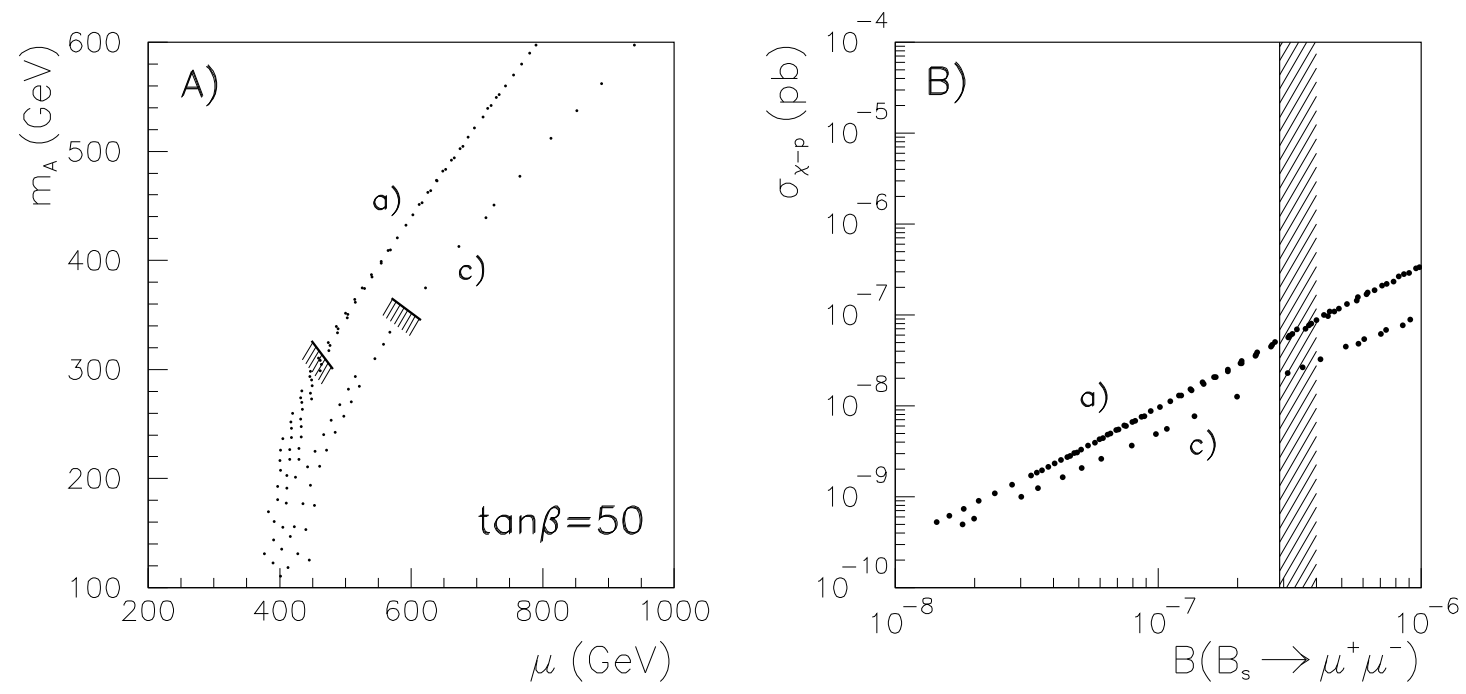

Figure 7: A) CP-odd mass versus the $\mu$ parameter for $\tan \beta=50, A=0$, and the two choices a) and c) in (3) for non-universal Higgses. All the points fulfil the experimental constraints and have the correct relic density. The constraint on $\mathrm{B}\left(B_{s}^{0} \rightarrow \mu^{+} \mu^{-}\right)$is displayed explicitly by means of a solid line with left shading which excludes the region below. B) Associated theoretical predictions for $\sigma_{\tilde{\chi}_{1}^{0}-p}$ versus $\mathrm{B}\left(B_{s}^{0} \rightarrow \mu^{+} \mu^{-}\right)$. The experimental limit on $\mathrm{B}\left(B_{s}^{0} \rightarrow \mu^{+} \mu^{-}\right)$is represented by the vertical line with right shading.

at the GUT scale decreases or increases with respect to its value in the universal case. Interestingly, we will find how certain choices of non-universal gauginos can help in decreasing $\mathrm{B}\left(B_{s}^{0} \rightarrow \mu^{+} \mu^{-}\right)$while allowing large values for $\sigma_{\tilde{\chi}_{1}^{0}-p}$.

\section{$2.1 \quad$ Decrease in $M_{3} / M_{1}\left(\delta_{3}^{\prime}<0\right)$}

When the ratio $M_{3} / M_{1}$ is decreased at the GUT scale (with $\delta_{3}^{\prime}<0$ ) heavier neutralinos, with a more important Higgsino composition, can be obtained. This is due to the reduction of the $\mu$ parameter through the contribution of $M_{3}$ to the RGE of $m_{H_{u}}^{2}$. These neutralinos can have a relatively high value for their direct detection cross section. Another consequence of the decrease in $M_{3} / M_{1}$ is the reduction in the value of the neutralino relic density, $\Omega_{\tilde{\chi}_{1}^{0}}$. This may be problematic, since the choices of non-universal scalars (3) already lead to a similar decrease, especially those where the Higgsino components of $\tilde{\chi}_{1}^{0}$ increase.

Interestingly, the variation in the gaugino mass parameters also has an influence on $\mathrm{B}\left(B_{s}^{0} \rightarrow \mu^{+} \mu^{-}\right)$. Namely, a decrease in $M_{3}$ leads to a smaller negative contribution in the RGE of $A_{t}$, thus raising its value at the electroweak scale and therefore effectively reducing the stop mixing and increasing the stop mass. This, together with the abovementioned reduction of $\mu$, entails a decrease in $\mathrm{B}\left(B_{s}^{0} \rightarrow \mu^{+} \mu^{-}\right)$, as we already explained 


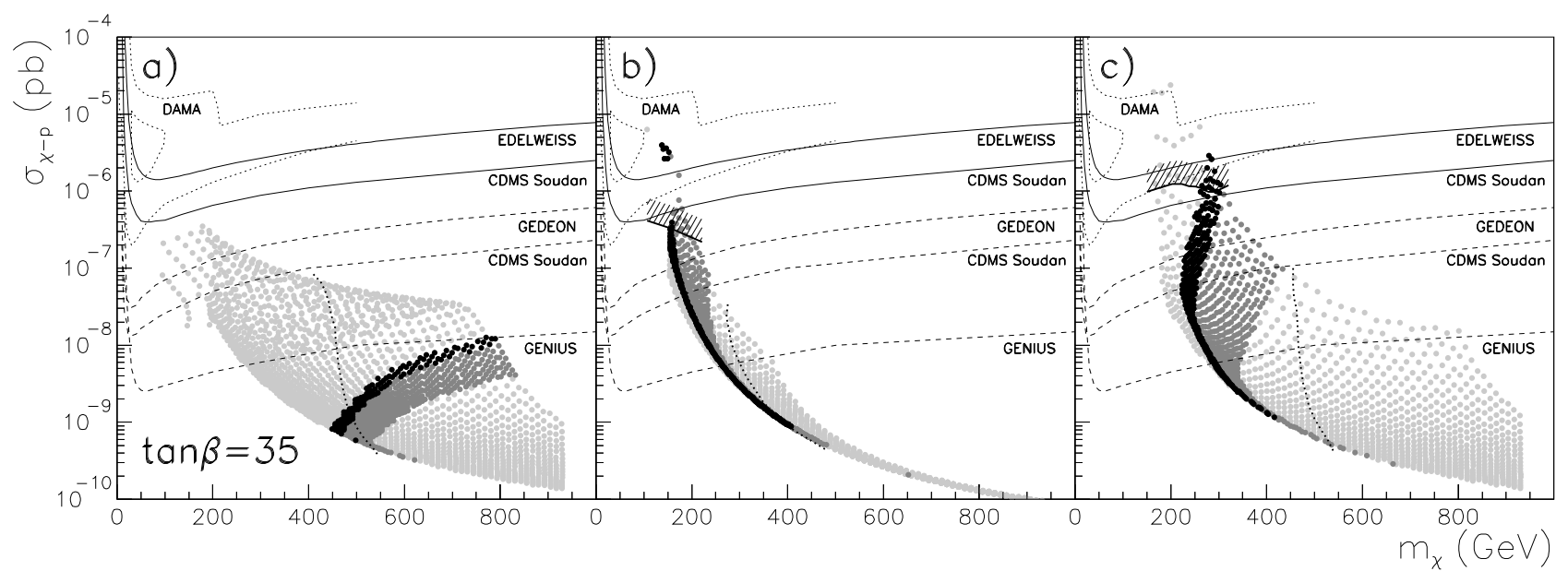

Figure 8: The same as Fig. 1 but for a case with $\delta_{2,3}^{\prime}=-0.25$.

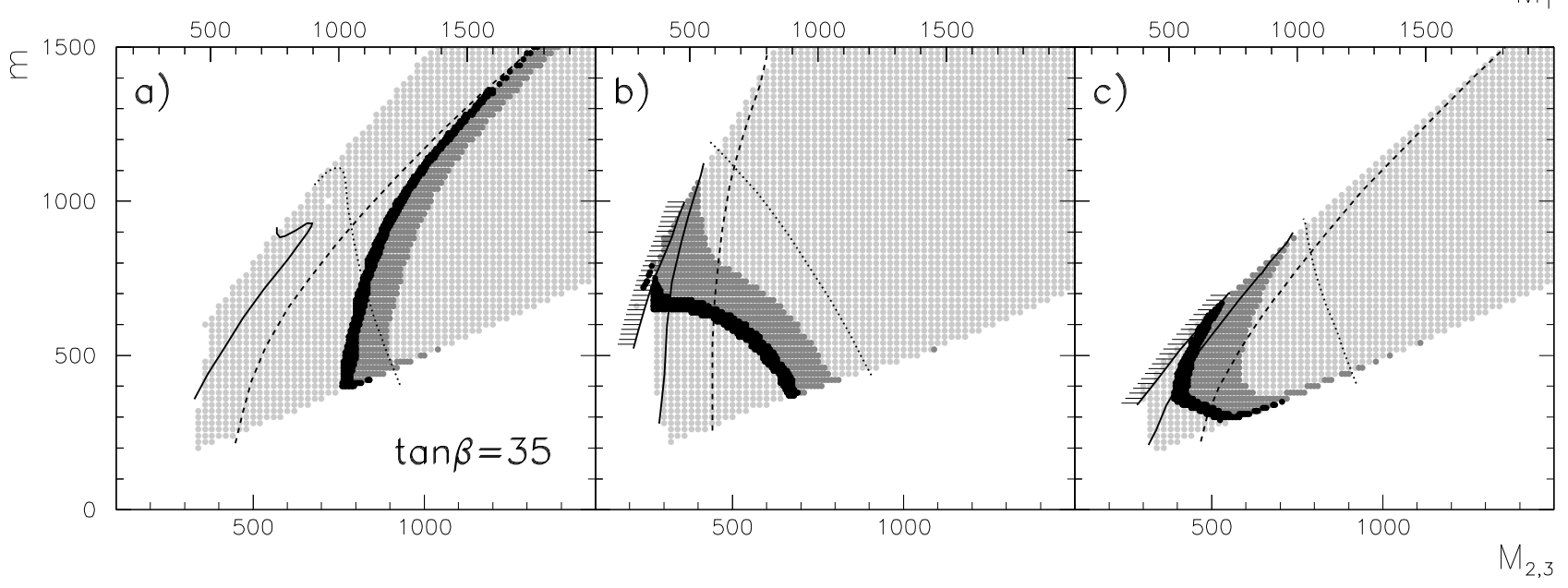

Figure 9: The same as Fig.2 but for a case with $\delta_{2,3}^{\prime}=-0.25$.

in the previous section.

Let us begin by considering an example with $\delta_{2,3}^{\prime}=-0.25$ in (22). In Fig. 8 the neutralino-proton cross section is plotted versus the neutralino mass, for $\tan \beta=35$, $A=0$, and a scan in $m$ and $M\left(m<1500 \mathrm{GeV}\right.$, and such that $50 \mathrm{GeV}<M_{3}<$ $1500 \mathrm{GeV}$ ) for the different choices of non-universal scalar parameters (3). Notice that, as a consequence of the decrease in the stop mixing and $\mu$ term, the experimental constraint in $\mathrm{B}\left(B_{s}^{0} \rightarrow \mu^{+} \mu^{-}\right)$is not as stringent as in the example with universal gauginos represented in Fig. (1. In particular, points very close or even within the present sensitivities of present dark matter detectors are obtained for cases b) and c), where $\sigma_{\tilde{\chi}_{1}^{0}-p} \lesssim 10^{-6} \mathrm{pb}$. In case a) the predictions for $\sigma_{\tilde{\chi}_{1}^{0}-p}$ are smaller due to the less effective reduction of the CP-odd Higgs mass. 


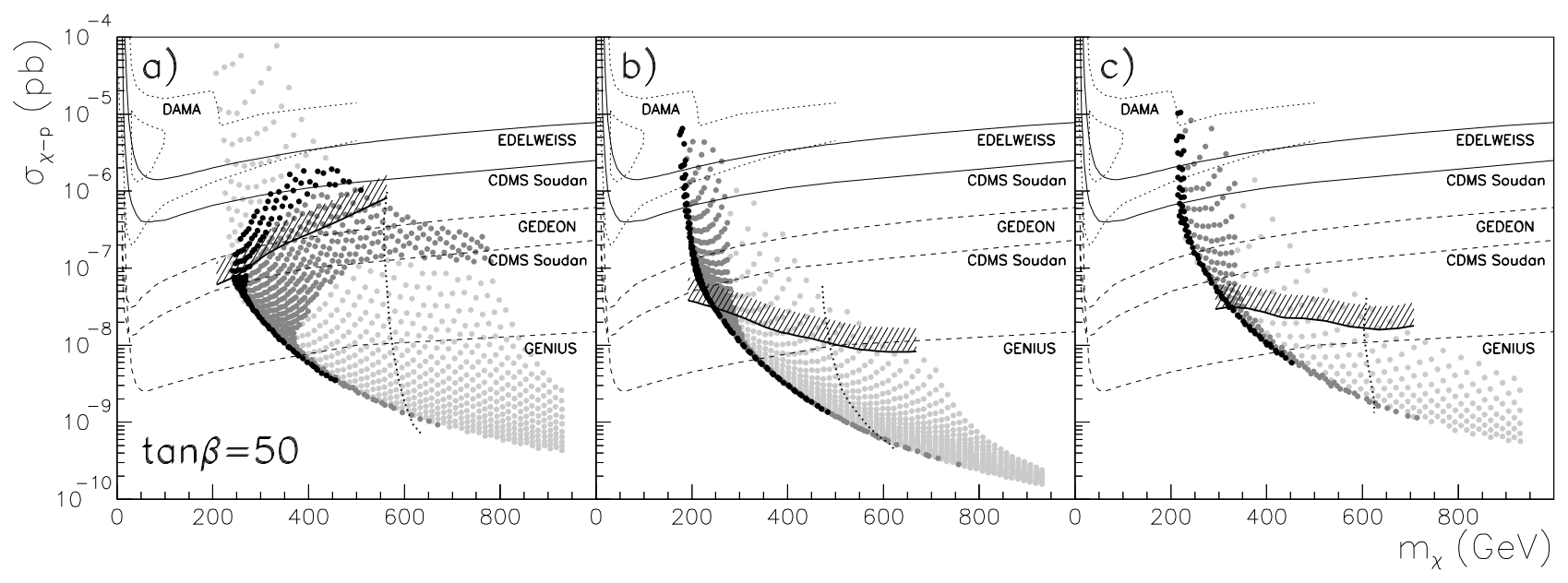

Figure 10: The same as Fig. 1 but for a case with $\tan \beta=50$ and $\delta_{2,3}^{\prime}=-0.25$.

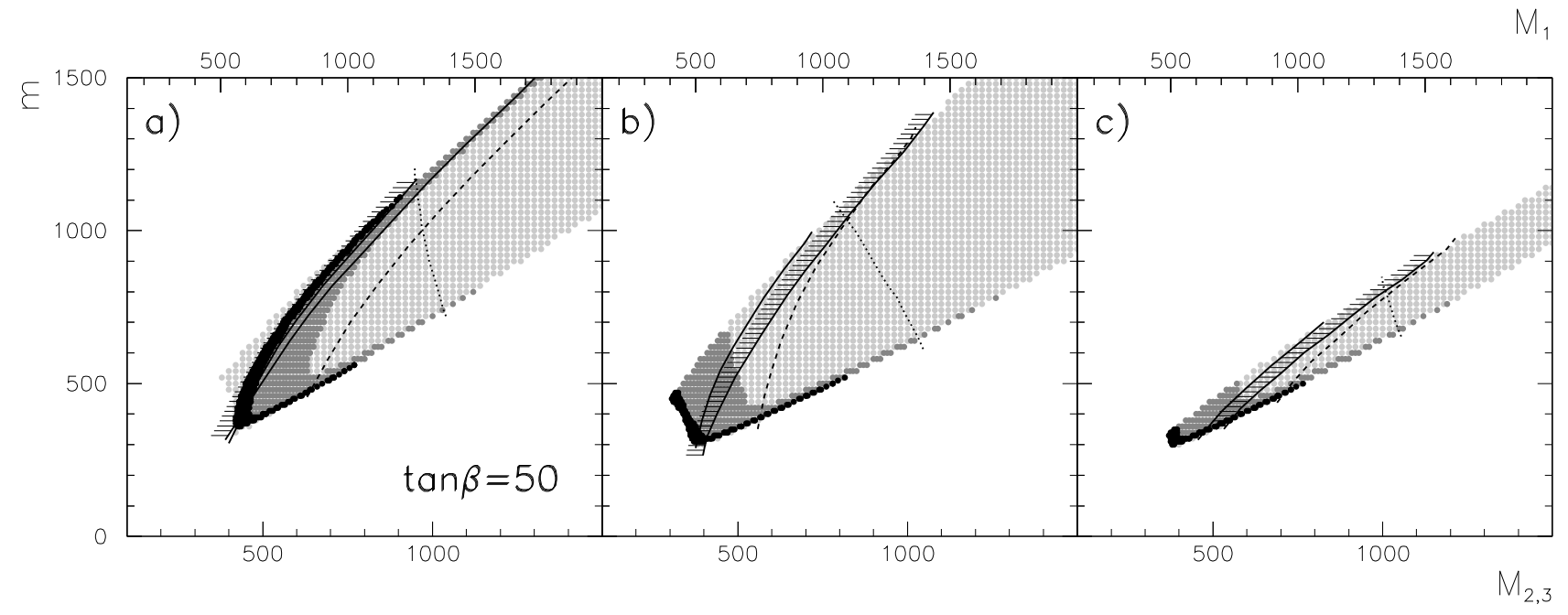

Figure 11: The same as Fig. 2 but for a case with $\tan \beta=50$ and $\delta_{2,3}^{\prime}=-0.25$.

The corresponding $\left(m, M_{i}\right)$ parameter space is displayed in Fig.9. Notice that all the experimentally viable points in case a) lie in a region which cannot be tested even by GENIUS. Contrariwise, in cases b) and c), CDMS Soudan and GENIUS will be able to test the regions with $M_{1} \lesssim 500 \mathrm{GeV}$ and $M_{1} \lesssim 700 \mathrm{GeV}$, respectively.

A further example, now with $\tan \beta=50$, is represented in Fig.10. Notice again how the $\mathrm{B}\left(B_{s}^{0} \rightarrow \mu^{+} \mu^{-}\right)$constraint is slightly less stringent than in the examples with universal gauginos of Fig. [3] For instance, points appear in case a) which could be tested in the future by the CDMS Soudan experiment with $m_{\tilde{\chi}_{1}^{0}} \approx 250 \mathrm{GeV}$. In cases b) and c) the detection cross section is bounded at $\sigma_{\tilde{\chi}_{1}^{0}-p} \lesssim 3 \times 10^{-8} \mathrm{pb}$, all the points lying below the predicted sensitivity of CDMS Soudan. 


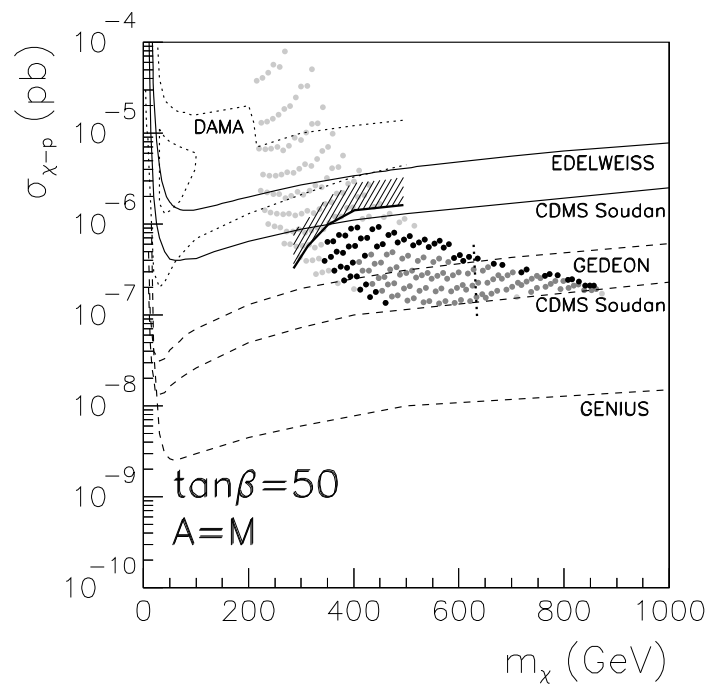

Figure 12: The same as Fig. 11 but for a case with $\tan \beta=50, A=M$, non-universal Higgs masses with $\delta_{1}=0$ and $\delta_{2}=1.5$, and non-universal gauginos with $\delta_{2,3}^{\prime}=-0.25$.

The related $\left(m, M_{i}\right)$ parameter space is represented in Fig.11. We find that the only experimentally allowed regions in the parameter space where the WMAP result for the relic density is obtained correspond to those in the coannihilation tail, close to the line where the stau becomes the LSP. As expected, the constraint on $\mathrm{B}\left(B_{s}^{0} \rightarrow \mu^{+} \mu^{-}\right)$ is responsible for the exclusion of those areas where the CP-odd Higgs mass is smaller.

Once more, the $\mathrm{B}\left(B_{s}^{0} \rightarrow \mu^{+} \mu^{-}\right)$constraint can be softened and large values for the neutralino direct detection cross section can be obtained by an appropriate choice of the initial parameters. As we commented in the previous section, a modification in the trilinear parameter such that it minimises the stop mixing and a small $\mu$ term can have this effect. Indeed, this possibility is now favoured by the fact that the decrease of $M_{3}$ contributes to both effects. To illustrate this we show in Fig.12] an example with $\tan \beta=50$ and non-universal gaugino masses with $\delta_{2,3}^{\prime}=-0.25$, where we have taken $A=M$, and non-universal Higgs masses with $\delta_{1}=0$ and $\delta_{2}=1.5$ in (11). We find how neutralinos fulfilling all experimental and astrophysical constraints appear with $\sigma_{\tilde{\chi}_{1}^{0}-p} \lesssim 10^{-6} \mathrm{pb}$ and $m_{\tilde{\chi}_{1}^{0}}=350-400 \mathrm{GeV}$. These neutralinos have a relatively large Higgsino composition and are very close to the present sensitivity of the CDMS Soudan detector.

\section{$2.2 \quad$ Increase in $M_{3} / M_{1}\left(\delta_{3}^{\prime}>0\right)$}

Increasing the value of $M_{3}$ with respect to $M_{1}$ at the GUT scale can be done with $\delta_{3}^{\prime}>0$ in (21). In this case, the constraint on the Higgs mass and on $b \rightarrow s \gamma$ will be satisfied 
for smaller values of $M$, and for this reason the effective value of $M_{1}$ can be smaller than in the universal case. Thus lighter neutralinos with a larger bino composition can be obtained.

Notice that in this case, being $M_{3}$ larger than in the universal case, it contributes more to the running of $A_{t}$. This means that the stop mixing is larger. Owing to this and to the slight increase in the $\mu$ term, we can expect larger values for $\mathrm{B}\left(B_{s}^{0} \rightarrow \mu^{+} \mu^{-}\right)$ and hence, a more stringent constraint.

The theoretical predictions for $\sigma_{\tilde{\chi}_{1}^{0}-p}$ as a function of the neutralino mass are represented in Fig.13 for an example with $\tan \beta=35, A=0$, the three choices for non-universal Higgses (3), and non-universal gaugino masses with $\delta_{2,3}^{\prime}=1$ in (2). As expected, lighter neutralinos than in those cases with $\delta_{3}^{\prime}<0$ shown in the previous section, are obtained. In the three cases the detection cross section is limited to be $\sigma_{\tilde{\chi}_{1}^{0}-p} \lesssim 10^{-7} \mathrm{pb}$, with neutralinos as light as $m_{\tilde{\chi}_{1}^{0}} \gtrsim 70 \mathrm{GeV}$. These fall within the projected sensitivities of GEDEON and CDMS Soudan. The experimental constraint on $\mathrm{B}\left(B_{s}^{0} \rightarrow \mu^{+} \mu^{-}\right)$plays no role in limiting the value of $\sigma_{\tilde{\chi}_{1}^{0}-p}$ of those points with the correct relic density.

The associated $\left(m, M_{i}\right)$ parameter space is depicted in Fig.14. The absence of an allowed region in the coannihilation tail in the three cases is remarkable. This is due to the occurrence of UFB directions in the Higgs potential in those areas with light stops. Because of the increase of $M_{3}$, the UFB constraints are more stringent and the coannihilation regions tend to become excluded [39]. Regarding the $\mathrm{B}\left(B_{s}^{0} \rightarrow \mu^{+} \mu^{-}\right)$ constraint, we find that the forbidden areas in cases b) and c) only lie in regions where the neutralino relic density is too low to reproduce the WMAP result. Concerning the potential reach of future dark matter detectors, the whole allowed area in case a) could be tested by GENIUS, while only those points with $M_{1} \lesssim 350 \mathrm{GeV}$ would be within the projected CDMS Soudan sensitivity. In cases b) and c) the area covered by CDMS Soudan corresponds to $M_{1} \lesssim 200 \mathrm{GeV}$ and $250 \mathrm{GeV}$, respectively. In case c) GENIUS could test points up to $M_{1} \lesssim 375 \mathrm{GeV}$.

Although for larger values of $\tan \beta$ one could expect a rise in $\sigma_{\tilde{\chi}_{1}^{0}-p}$, the associated increment in $\mathrm{B}\left(B_{s}^{0} \rightarrow \mu^{+} \mu^{-}\right)$renders this possibility very constrained. For instance, the predictions for the neutralino detection cross section are represented in Fig. [15] for the former example but taking $\tan \beta=50$. Although points with very large $\sigma_{\tilde{\chi}_{1}^{0}-p}$ appear, all of these have a too large $\mathrm{B}\left(B_{s}^{0} \rightarrow \mu^{+} \mu^{-}\right)$and are ruled out. In the end, $\sigma_{\tilde{\chi}_{1}^{0}-p} \lesssim 2 \times 10^{-8} \mathrm{pb}$ in case a) and $\sigma_{\tilde{\chi}_{1}^{0}-p} \lesssim 10^{-8} \mathrm{pb}$ in cases b) and c), beyond the reach of the projected CDMS Soudan detector. Notice that these limits are slightly lower than in the case represented in Fig.10, where $\delta_{3}^{\prime}<0$. As we commented above, this is due to the influence of $M_{3}$ in the stop mixing and $\mu$ term.

Only GENIUS would be able to test those regions with $m_{\tilde{\chi}_{1}^{0}} \lesssim 250 \mathrm{GeV}$ in case a) and $m_{\tilde{\chi}_{1}^{0}} \lesssim 200 \mathrm{GeV}$ in b) and c). As we see in Fig.16, where the associated $\left(m, M_{i}\right)$ parameter is plotted, all the allowed points correspond to the regions where, due to 


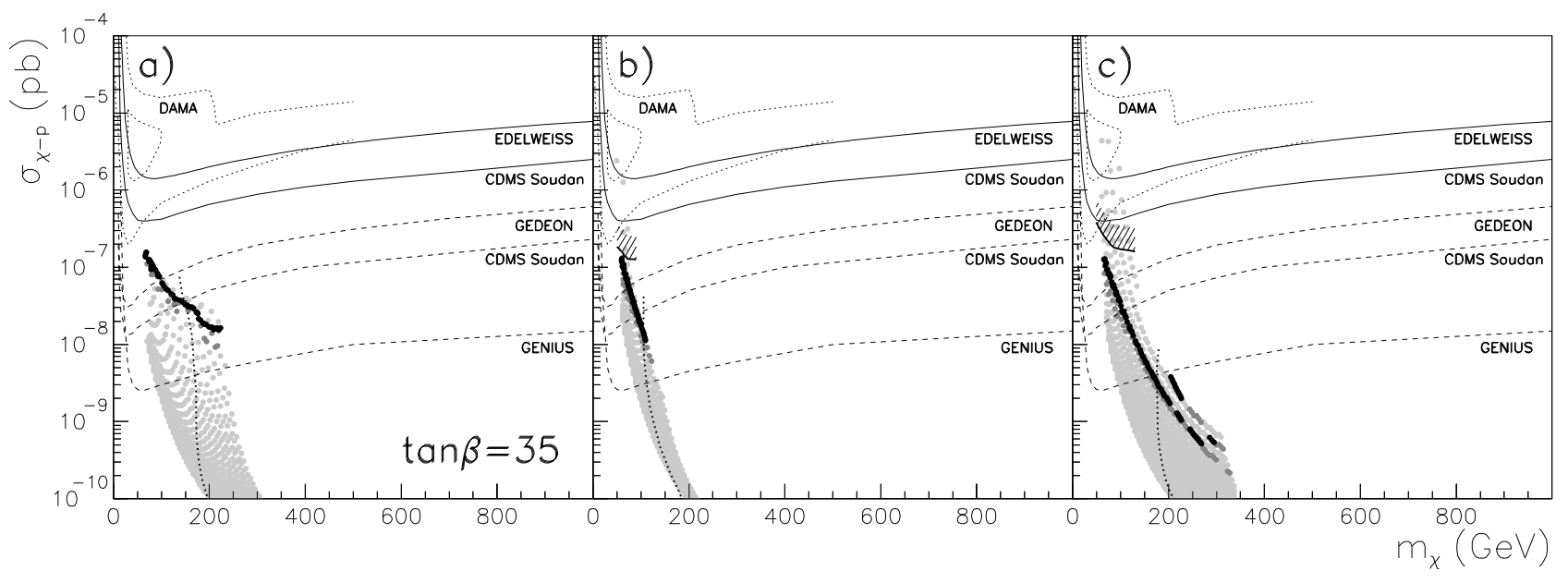

Figure 13: The same as Fig. 1] but for a case with $\delta_{2,3}^{\prime}=1$.

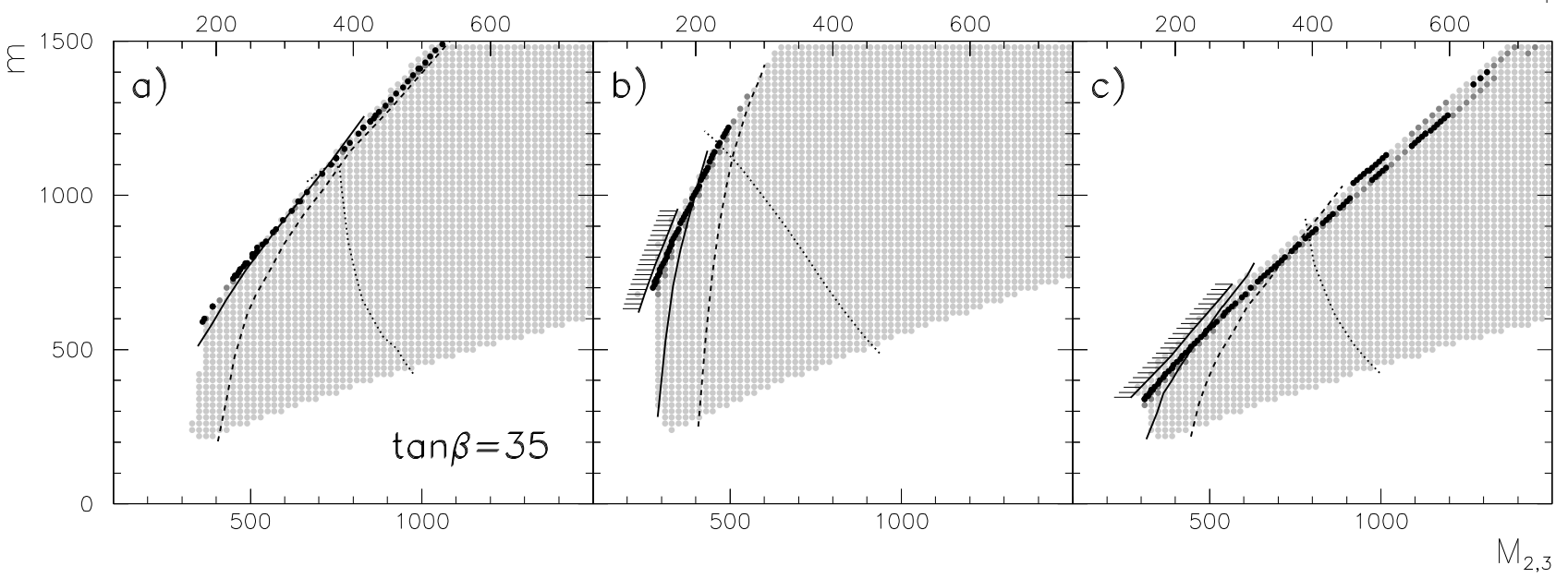

Figure 14: The same as Fig.2 but for a case with $\delta_{2,3}^{\prime}=1$. 


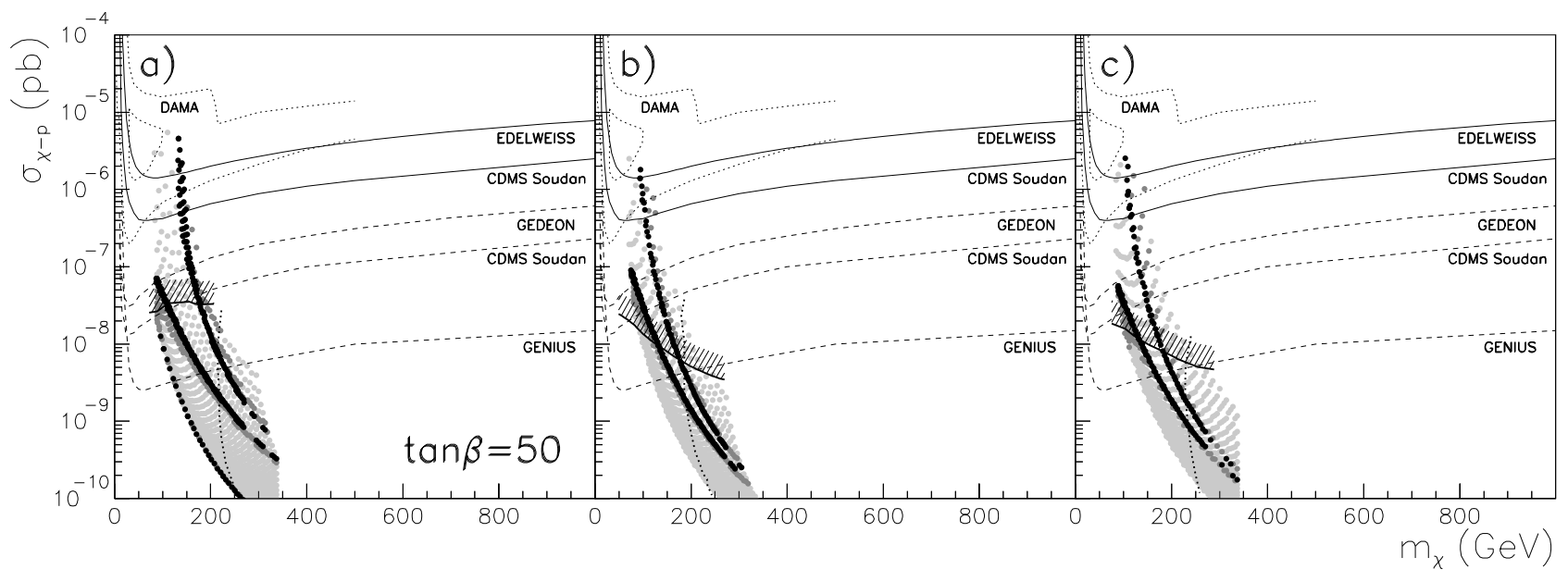

Figure 15: The same as Fig. 1 but for a case with $\tan \beta=50$ and $\delta_{2,3}^{\prime}=1$.

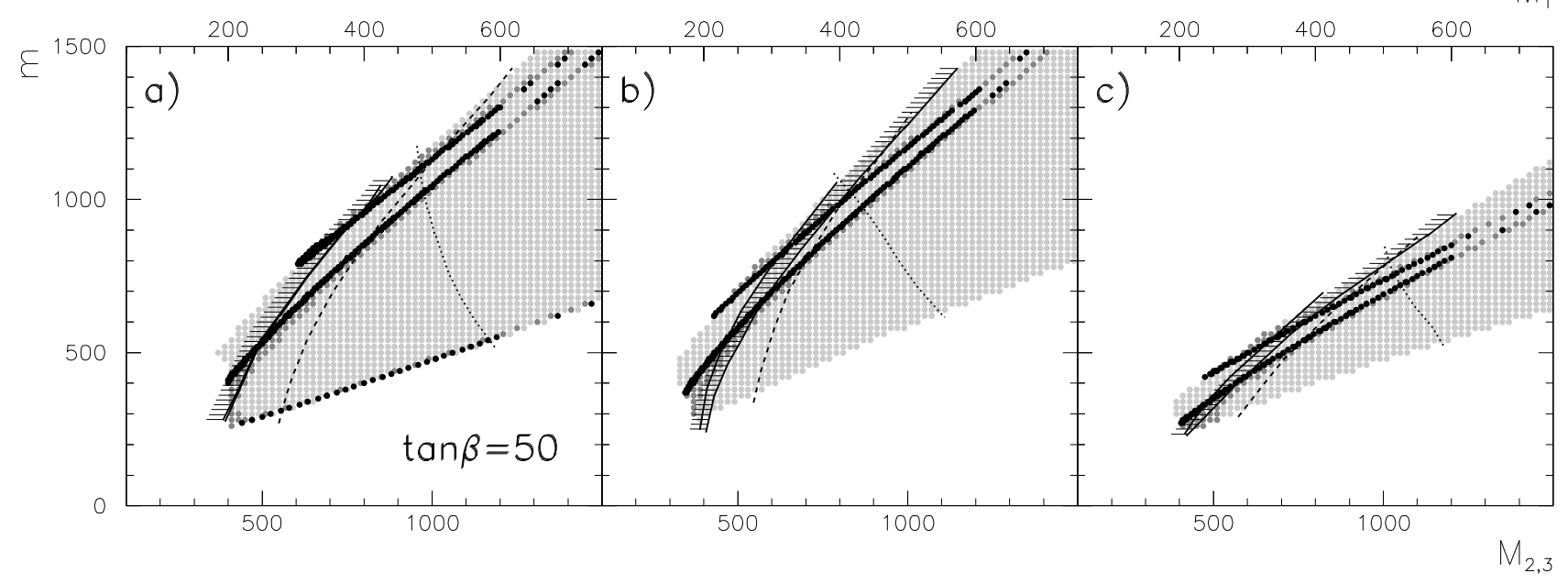

Figure 16: The same as Fig. 2 but for a case with $\tan \beta=50$ and $\delta_{2,3}^{\prime}=1$. 


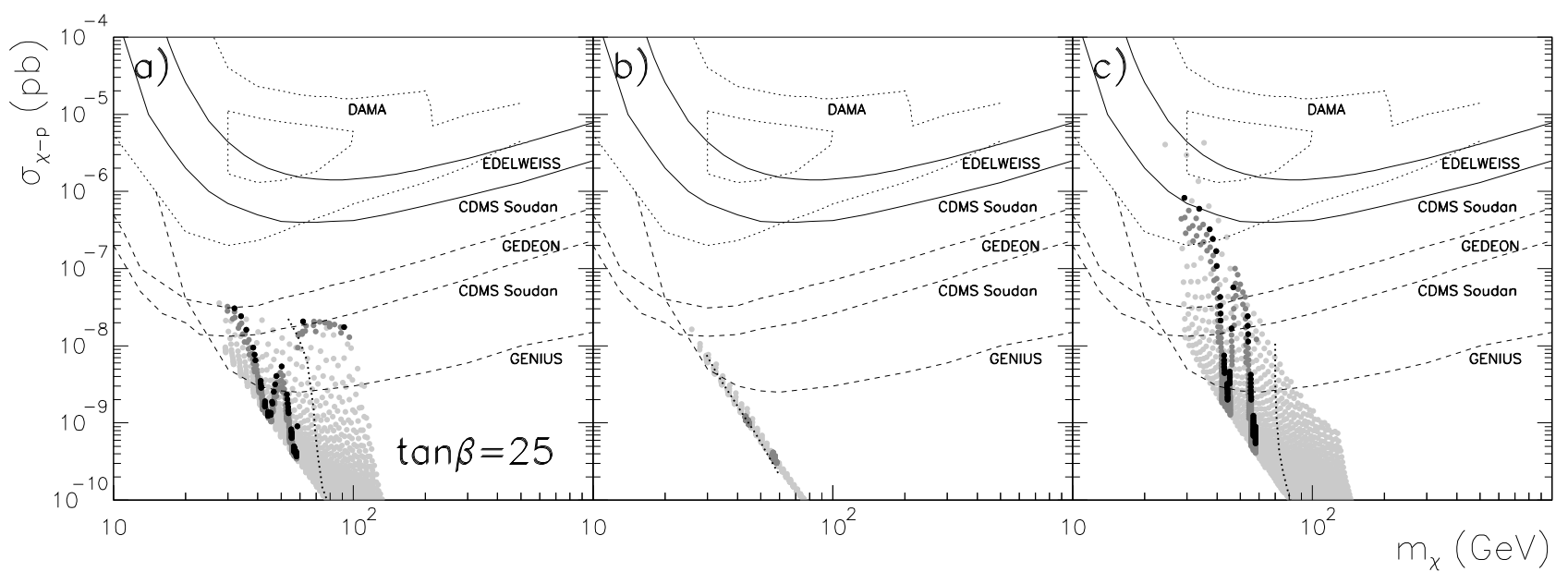

Figure 17: The same as Fig. 1 but for a case with $\tan \beta=25$ and $\delta_{2,3}^{\prime}=3$.

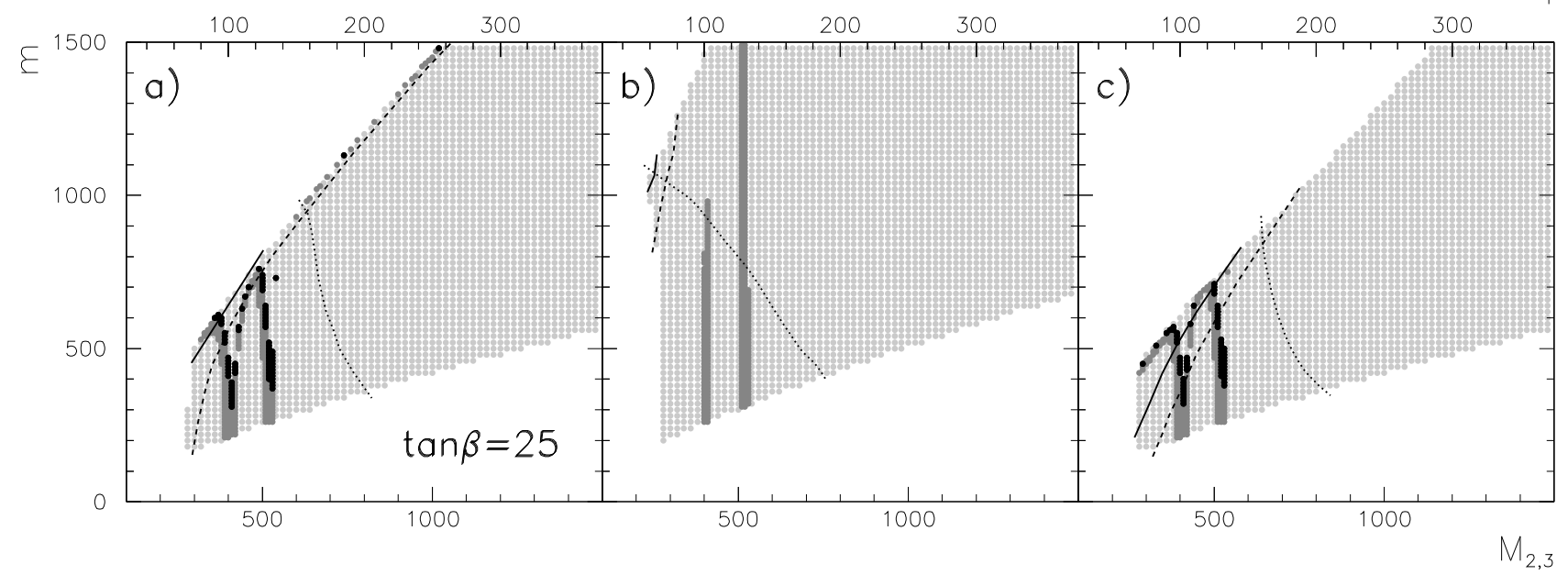

Figure 18: The same as Fig. [2] but for a case with $\tan \beta=25$ and $\delta_{2,3}^{\prime}=3$.

resonant annihilation of neutralinos mediated by the CP-odd Higgs, the correct relic density is obtained. This happens when $2 m_{\tilde{\chi}_{1}^{0}} \approx m_{A}$. Only case a) presents some allowed points in the coannihilation tail.

A further reduction in the neutralino mass is possible if larger values are taken for $\delta_{2,3}^{\prime}$. Obviously, the bino composition of these neutralinos also increases. Figure 17 illustrates this possibility for an example with $\delta_{2,3}^{\prime}=3, \tan \beta=25$, and $A=0$. Neutralinos as light as $m_{\tilde{\chi}_{1}^{0}} \approx 30 \mathrm{GeV}$ appear with large values for the detection cross section, $\sigma_{\tilde{\chi}_{1}^{0}-p} \approx 10^{-6} \mathrm{pb}$. Interestingly, despite the lightness of the Higgs masses that is necessary, due to the moderate values of $\tan \beta$ these points are not in contradiction with the experimental bound on $\mathrm{B}\left(B_{s}^{0} \rightarrow \mu^{+} \mu^{-}\right)$. 


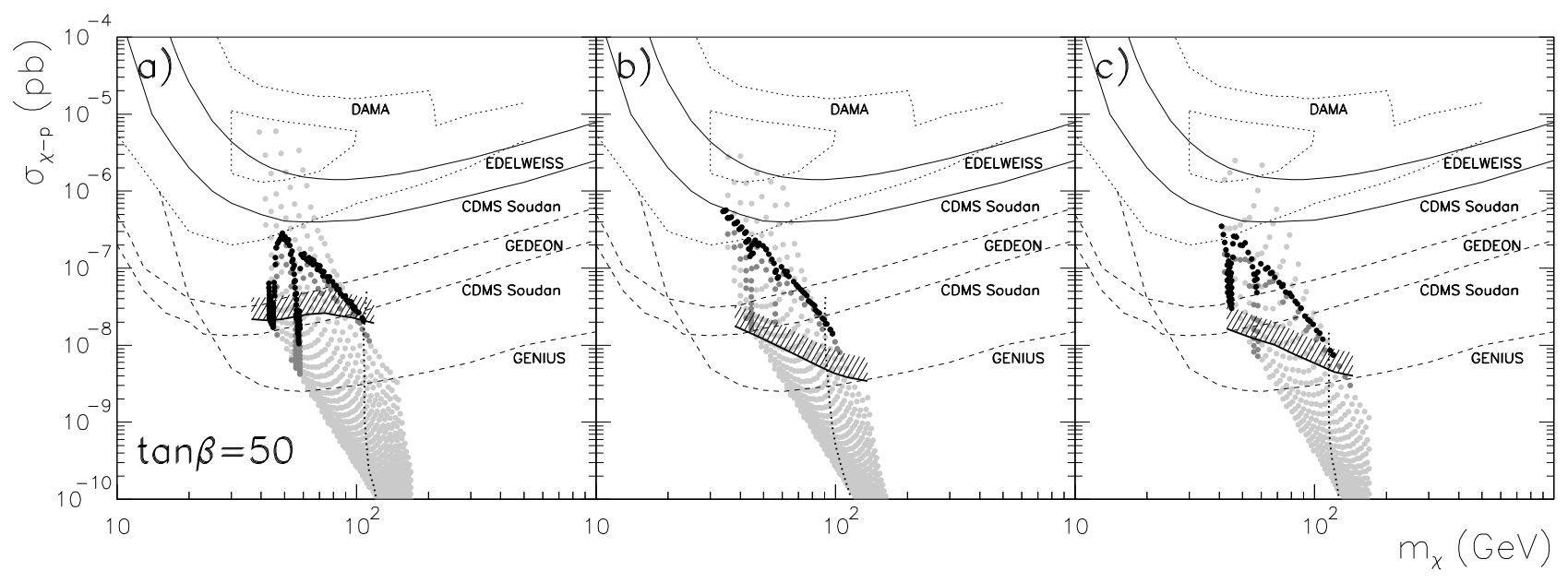

Figure 19: The same as Fig. 1 but for a case with $\tan \beta=50$ and $\delta_{2,3}^{\prime}=3$.

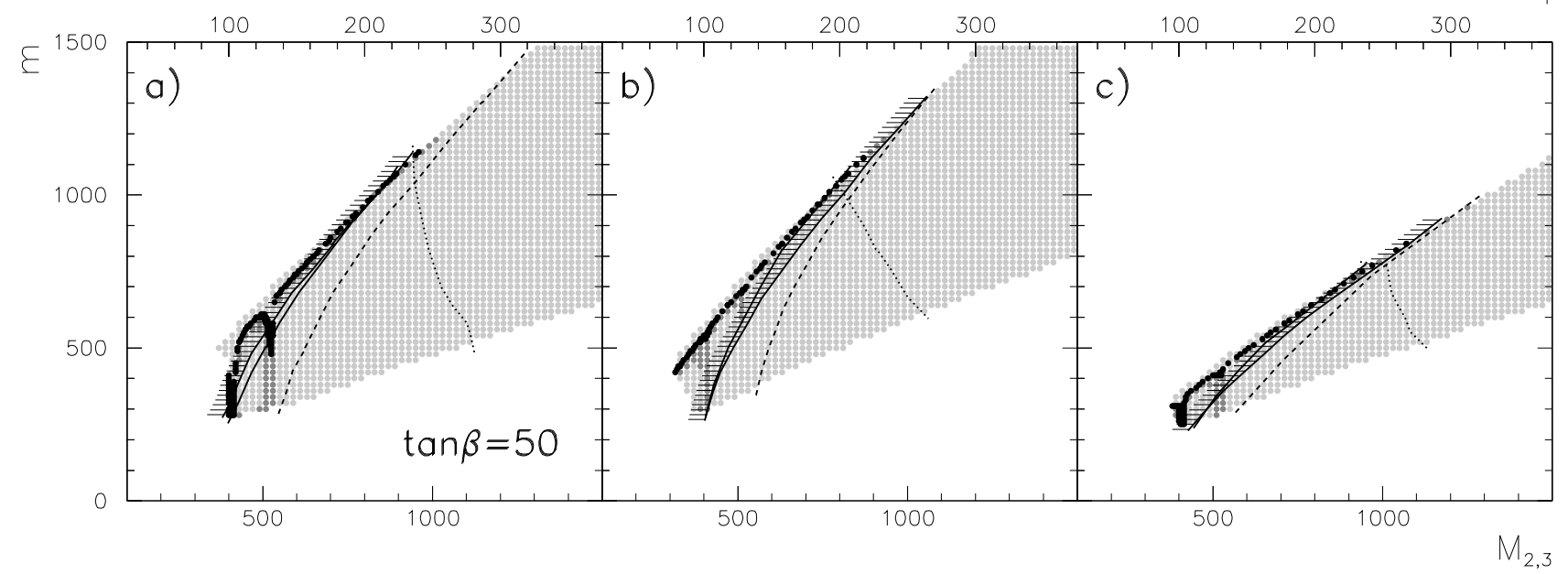

Figure 20: The same as Fig. [2] but for a case with $\tan \beta=50$ and $\delta_{2,3}^{\prime}=3$.

Nevertheless, these cases disappear as soon as $\tan \beta \gtrsim 30$, in which case the only regions of the parameter space for which all the constraints are fulfilled coincide with the $s$-channel resonant annihilation of neutralinos mediated by the lightest Higgs or the $Z$ boson, occurring when $m_{\tilde{\chi}_{1}^{0}} \approx m_{h, Z} / 2$. For instance, the case with $\tan \beta=50$ is represented in Fig. 19, The corresponding $\left(m, M_{i}\right)$ parameter space is shown in Fig. 20, It can be seen how the combination of the UFB and $\mathrm{B}\left(B_{s}^{0} \rightarrow \mu^{+} \mu^{-}\right)$constraints leave only the extremely fine-tuned chimneys corresponding to resonant neutralino annihilation. 


\subsubsection{Very light neutralinos}

Very light neutralinos can be obtained with non-universal gaugino masses when $M_{1} \ll$ $M_{2}, M_{3}, \mu$ (thus $\tilde{\chi}_{1}^{0}$ being bino-like) and when the CP-odd Higgs is light enough $\left(m_{A} \lesssim 200 \mathrm{GeV}\right)$ in order to enhance neutralino annihilation and obtain a relic density compatible with WMAP. This can be achieved with the choices of Higgs nonuniversalities we presented in (3) for $\tan \beta \gtrsim 35$. Neutralinos obtained in this way can be as light as $10 \mathrm{GeV}$ [39], and have a cross section within the reach of future dark matter experiments.

However, due to the smallness of the Higgs masses and large values of $\tan \beta$, these regions of the parameter space are likely to imply sizable $\mathrm{B}\left(B_{s}^{0} \rightarrow \mu^{+} \mu^{-}\right)$, and thus be very constrained by its improved experimental result.

As an example, we show in Fig.21 the case with $\delta_{2,3}^{\prime}=10$ and $\tan \beta=50$ for the three different choices of Higgs masses (33). Neutralinos fulfilling the experimental constraints and with a relic density in agreement with WMAP appear with $m_{\tilde{\chi}_{1}^{0}} \gtrsim 15 \mathrm{GeV}$ and with very large values for the cross section, $\sigma_{\tilde{\chi}_{1}^{0}-p} \gtrsim 10^{-6} \mathrm{pb}$. Unfortunately, once the experimental constraint on $\mathrm{B}\left(B_{s}^{0} \rightarrow \mu^{+} \mu^{-}\right)$is applied, all these cases are ruled out. This is also shown in Fig. 22, where the $\left(m, M_{i}\right)$ parameter space is depicted. All the points that have the correct relic density appear in a narrow line which runs alongside the region where the CP-odd Higgs mass becomes tachyonic. This is contained within the area excluded by the stringent $\mathrm{B}\left(B_{s}^{0} \rightarrow \mu^{+} \mu^{-}\right)$constraint.

Although in principle it could be possible to escape the drastic limitations imposed by $\mathrm{B}\left(B_{s}^{0} \rightarrow \mu^{+} \mu^{-}\right)$by choosing suitable input parameters (which lead to a decrease of the stop mixing and $\mu$ parameter), the amount of fine tuning which is needed is huge. Besides, as already mentioned, the CP-odd Higgs has to remain very light to reproduce the correct value for the relic density. As a consequence, in order to induce a sufficient decrease on the ratio $\left(\mu A_{t} / m_{\tilde{t}_{L}}^{2}\right)^{2}$ one needs such a small value of $\mu$ that the constraint on the chargino mass and on $b \rightarrow s \gamma$ become difficult to fulfil. Also decreasing $A_{t} / m_{\tilde{t}_{L}}$ is problematic since it entails a decrease of the Higgs mass and we are already very close to its experimental limit.

\section{Conclusions}

In this paper we have performed an analysis of the direct detection of neutralino dark matter in general supergravity scenarios, where non-universality of soft scalar and gaugino masses can be present, in the light of the recent experimental constraint on $\mathrm{B}\left(B_{s}^{0} \rightarrow \mu^{+} \mu^{-}\right)$from CDF and D0 Collaborations at the Tevatron. More specifically, we have computed the theoretical predictions for the spin-independent neutralino-nucleon cross section, $\sigma_{\tilde{\chi}_{1}^{0}-p}$, and compared it with the sensitivities of present and future dark 


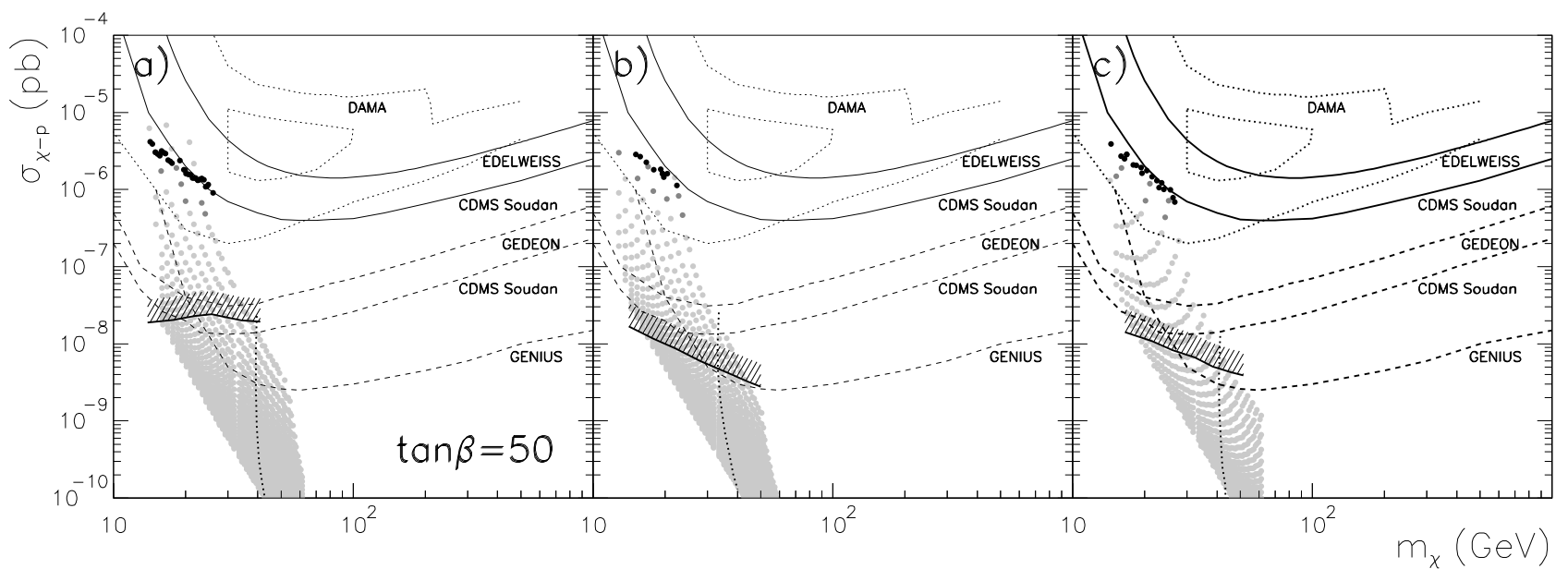

Figure 21: The same as in Fig.1 but with non-universal gauginos with, $\delta_{2,3}^{\prime}=10$, and $\tan \beta=50$.

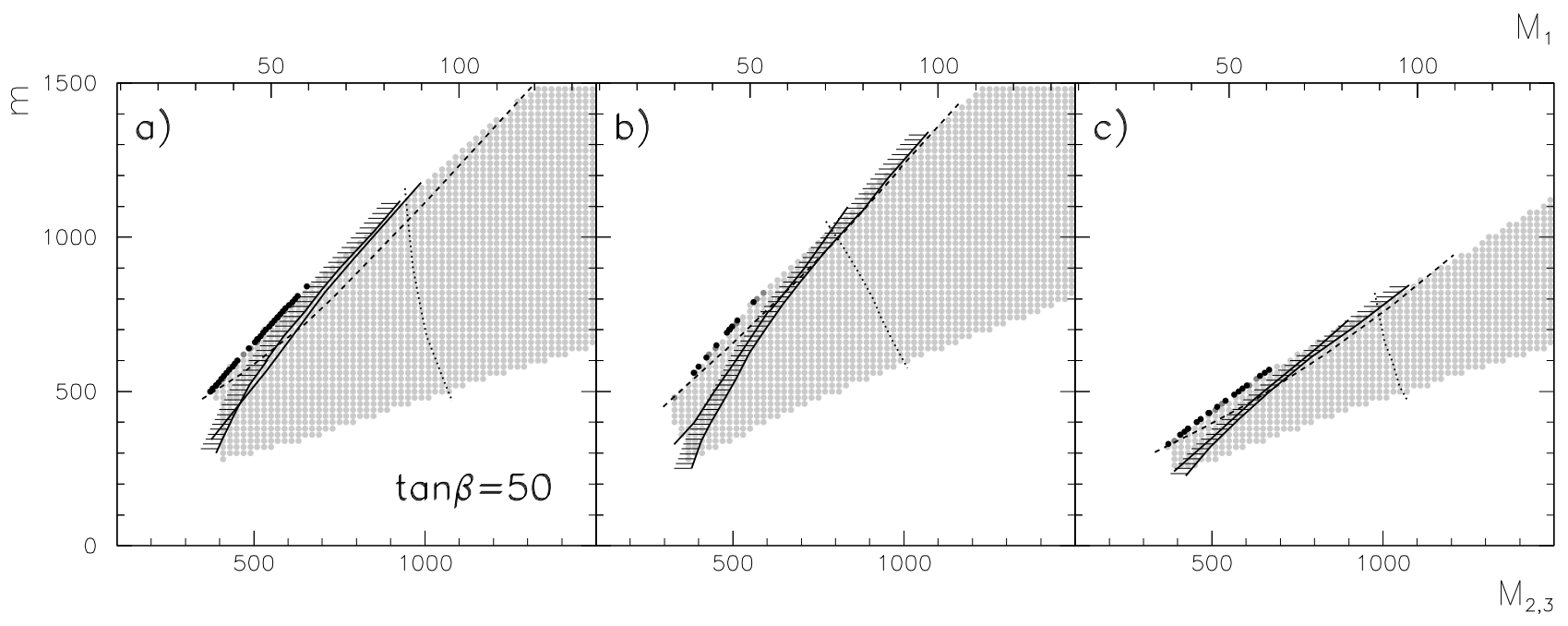

Figure 22: The same as Fig. [2 but for a case with $\tan \beta=50$ and $\delta_{2,3}^{\prime}=10$.

matter detectors.

We find that the $\mathrm{B}\left(B_{s}^{0} \rightarrow \mu^{+} \mu^{-}\right)$puts very strong limitations on $\sigma_{\tilde{\chi}_{1}^{0}-p}$ in many interesting cases of non-universal scalar and gaugino masses. Both observables are enhanced in the large $\tan \beta$ limit due to the neutral Higgs exchange diagrams. Therefore, although the neutralino detection cross section could be enhanced up to $\sigma_{\tilde{\chi}_{1}^{0}-p} \sim 10^{-5} \mathrm{pb}$, those regions of the parameter space where the DAMA and the CDMS experiments could detect signals are mostly excluded once the $\mathrm{B}\left(B_{s}^{0} \rightarrow \mu^{+} \mu^{-}\right)$constraint is taken into account. As a consequence, the resulting cross section typically becomes quite small, at the level of $10^{-7} \mathrm{pb}$ or even less. 
Nevertheless, it is not impossible to evade this constraint and obtain large neutralino detection cross sections. The correlation between $\mathrm{B}\left(B_{s}^{0} \rightarrow \mu^{+} \mu^{-}\right)$and $\sigma_{\tilde{\chi}_{1}^{0}-p}$ can be diluted if the top trilinear parameter is tuned at the GUT scale and/or the $\mu$ parameter decreased in order to reduce the stop mixing and induce a smaller chargino mediated $b \rightarrow s$ transition.

Some choices of scalar and gaugino non-universalities favour these effects. In particular, we have shown that when the gluino mass parameter, $M_{3}$, decreases with respect to the bino mass term, $M_{1}$, heavier neutralinos with a larger Higgsino composition can be obtained. In this case the $\mathrm{B}\left(B_{s}^{0} \rightarrow \mu^{+} \mu^{-}\right)$constraint is softer and, when Higgs non-universalities are also considered, $\sigma_{\tilde{\chi}_{1}^{0}-p} \gtrsim 10^{-6} \mathrm{pb}$ can be obtained.

On the other hand, light bino-like neutralinos, which appear when $M_{3}$ increases with respect to $M_{1}$ are more difficult to reconcile with the experimental constraint on $\mathrm{B}\left(B_{s}^{0} \rightarrow \mu^{+} \mu^{-}\right)$. Still, neutralinos as light as $m_{\tilde{\chi}_{1}^{0}} \gtrsim 30 \mathrm{GeV}$ and with $\sigma_{\tilde{\chi}_{1}^{0}-p} \lesssim 10^{6} \mathrm{pb}$, within the DAMA region, can be obtained with moderate values of $\tan \beta$.

\section{Acknowledgements}

The work of SB was supported by NSERC of Canada. DGC gratefully acknowledges financial support from the PPARC and from the European Network for Theoretical Astroparticle Physics (ENTApP), member of ILIAS, EC contract number RII-CT2004-506222. YGK was supported by the Korean Federation of Science and Technology Societies through the Brain Pool program. PK was supported in part by KOSEF Sundo grant R02-2003-000-10085-0, and KOSEF through CHEP at Kyungpook National University. The work of CM was supported in part by the Spanish DGI del MEC under Acción Integrada Hispano-Alemana HA2002-0117, and under Proyectos Nacionales BFM2003-01266 and FPA2003-04597, and also by the European Union under the RTN program MRTN-CT-2004-503369, and under the ENTApP Network of the ILIAS project RII3-CT-2004-506222.

DGC would like to thank A. Dedes for useful discussions.

\section{References}

[1] For a recent review, see C. Muñoz, 'Dark matter detection in the light of recent experimental results', Int. J. Mod. Phys. A19 (2004) 2093 arXiv:hep-ph/0309346.

[2] For reviews, see e.g. M. Persic, P. Salucci and F. Stel, 'The universal rotation curve of spiral galaxies: I. The dark matter connection', Mon. Not. Roy. Astron. Soc. 281 (1996) 27 arXiv:astro-ph/9506004; 
Y. Sofue and V. Rubin, 'Rotation curves of spiral galaxies', arXiv:astro-ph/0010594,

M. Roncadelli, 'Searching for dark matter', arXiv:astro-ph/0307115;

P. Salucci and M. Persic, 'Dark matter halos around galaxies', arXiv:astro-ph/9703027;

E. Battaner and E. Florido, 'The rotation curve of spiral galaxies and its cosmological implications', Fund. Cosmic Phys. 21 (2000) 1 arXiv:astro-ph/0010475.

[3] For a review, see W. L. Freedman, 'Determination of cosmological parameters', Phys. Scripta T85 (2000) 37 arXiv:astro-ph/9905222.

[4] WMAP Collaboration, C. L. Bennett et al., 'First year Wilkinson microwave anisotropy probe (WMAP) observations: preliminary maps and basic results', Astrophys. J. Suppl. 148 (2003) 1 arXiv:astro-ph/0302207; D. N. Spergel et al., 'First year Wilkinson microwave anisotropy probe (WMAP) observations: determination of cosmological parameters', Astrophys. J. Suppl. 148 (2003) 175 arXiv:astro-ph/0302209; L. Verde et al., 'First year Wilkinson microwave anisotropy probe (WMAP) observations: parameter estimation methodology', Astrophys. J. Suppl. 148 (2003) 195 arXiv:astro-ph/0302218.

[5] DAMA Collaboration, R. Bernabei et al., 'Search for WIMP annual modulation signature: results from DAMA/NaI-3 and DAMA/NaI-4 and the global combined analysis', Phys. Lett. B 480 (2000) 23; 'Dark matter search', Riv. Nuovo Cim. 26 (2003) 1 arXiv:astro-ph/0307403.

[6] P. Belli, R. Cerulli, N. Fornengo and S. Scopel, 'Effect of the galactic halo modeling on the DAMA/NaI annual modulation result: an extended analysis of the data for WIMPs with a purely spin-independent coupling', Phys. Rev. D 66 (2002) 043503 arXiv:hep-ph/0203242.

[7] CDMS Collaboration, D. S. Akerib et al., 'First results from the cryogenic dark matter search in the Soudan underground lab', Phys. Rev. Lett. 93 (2004) 211301 arXiv:astro-ph/0405033.

[8] EDELWEISS Collaboration, A. Benoit et al., 'First results of the EDELWEISS WIMP search using a 320-g heat-and-ionization Ge detector', Phys. Lett. B513 (2001) 15 arXiv:astro-ph/0106094; 'Improved exclusion limits from the EDELWEISS WIMP search', Phys. Lett. B545 (2002) 43 |arXiv:astro-ph/0206271.

[9] ZEPLIN I Collaboration, N.J.T. Smith et al., 'Current status of dark matter searches at the Boulby facility', talk given at TAUP 2003, Seattle, Washington.

[10] A. Morales, 'Searching for WIMP dark matter: the case for Germanium ionization detectors', arXiv:hep-ex/0111089. 
[11] GENIUS Collaboration, H. V. Klapdor-Kleingrothaus et al., 'GENIUS - a Supersensitive Germanium Detector System for Rare Events', arXiv:hep-ph/9910205.

[12] See e.g., E. Aprile et al., 'The XENON dark matter search experiment', Nucl. Phys. Proc. Suppl. 138 (2005) 156 arXiv:astro-ph/0407575.

[13] D. G. Cerdeño, E. Gabrielli, M. E. Gomez, and C. Muñoz, 'Neutralino nucleon cross section and charge and colour breaking constraints', J. High Energy Phys. 06 (2003) 030 arXiv:hep-ph/0304115.

[14] CDF Collaboration, D. Acosta et al., 'Search for $B_{s}^{0} \rightarrow \mu^{+} \mu^{-}$and $B_{d}^{0} \rightarrow \mu^{+} \mu^{-}$ decays in $p \bar{p}$ collisions at $\sqrt{s}=1.96$ TeV', Phys. Rev. Lett. 93 (2004) 032001 arXiv:hep-ex/0403032.

[15] D0 Collaboration, V. M. Abazov et al. 'A search for the flavor-changing neutral current decay $B_{s}^{0} \rightarrow \mu^{+} \mu^{-}$in $p \bar{p}$ collisions at $\sqrt{s}=1.96 \mathrm{TeV}$ with the D0 detector', Phys. Rev. Lett. 94 (2005) 071802 arXiv:hep-ex/0410039.

[16] S. Baek, Y. G. Kim and P. Ko, 'Neutralino dark matter scattering and $B_{s} \rightarrow \mu^{+} \mu^{-}$ in SUSY models', J. High Energy Phys. 02 (2005) 067 arXiv:hep-ph/0406033.

[17] R. Arnowitt, B. Dutta, T. Kamon and M. Tanaka, 'Detection of $B_{s} \rightarrow \mu^{+} \mu^{-}$at the Tevatron Run II and constraints on the SUSY parameter space', Phys. Lett. $B 538$ (2002) 121 arXiv:hep-ph/0203069.

[18] J. R. Ellis, K. A. Olive and V. C. Spanos, 'On the Interpretation of $B_{s} \rightarrow \mu^{+} \mu^{-}$ in the CMSSM', arXiv:hep-ph/0504196.

[19] V. Berezinsky, A. Bottino, J. Ellis, N.Fornengo, G. Mignola and S. Scopel, 'Neutralino dark matter in supersymmetric models with non-universal scalar mass terms', Astropart. Phys. 5 (1996) 1 arXiv:hep-ph/9508249.

[20] R. Arnowitt and P. Nath, 'Non-universal soft SUSY breaking and dark matter', Phys. Rev. D 56 (1997) 2820 arXiv:hep-ph/9701301.

[21] A. Bottino, F. Donato, N. Fornengo and S. Scopel, 'Compatibility of the new DAMA/NaI data on an annual modulation effect in WIMP direct search with a relic neutralino in supergravity schemes', Phys. Rev. D 59 (1999) 095004 arXiv:hep-ph/9808459.

[22] R. Arnowitt and P. Nath, 'Annual modulation signature for the direct detection of Milky Way Wimps and supergravity models', Phys. Rev. D 60 (1999) 044002 arXiv:hep-ph/9902237.

[23] E. Accomando, R. Arnowitt, B. Dutta and Y. Santoso, 'Neutralino proton cross sections in supergravity models', Nucl. Phys. B 585 (2000) 124 arXiv:hep-ph/0001019. 
[24] J. Ellis, A. Ferstl and K. A. Olive, 'Exploration of elastic scattering rates for supersymmetric dark matter', Phys. Rev. D 63 (2001) 065016 arXiv:hep-ph/0007113;

J. Ellis, K. A. Olive and Y. Santoso, 'The MSSM parameter space with nonuniversal Higgs masses', Phys. Lett. B 539 (2002) 107 arXiv:hep-ph/0204192;

J. Ellis, T. Falk, K. A. Olive and Y. Santoso, 'Exploration of the MSSM with nonuniversal Higgs masses', Nucl. Phys. B 652 (2003) 259 arXiv:hep-ph/0210205;

J. Ellis, A. Ferstl, K. A. Olive and Y. Santoso, 'Direct detection of dark matter in the MSSM with nonuniversal Higgs masses', Phys. Rev. D 67 (2003) 123502 arXiv:hep-ph/0302032;

J. Ellis, K. A. Olive, Y. Santoso and V. C. Spanos, 'Update on the direct detection of supersymmetric dark matter', arXiv:hep-ph/0502001.

[25] M. Drees, Y. G. Kim, T. Kobayashi and M. M. Nojiri, 'Direct detection of neutralino dark matter and the anomalous dipole moment of the muon', Phys. Rev. D 63 (2001) 115009 arXiv:hep-ph/0011359.

[26] Y. G. Kim and M. M. Nojiri, 'Implications of muon anomalous magnetic moment for direc detection of neutralino dark matter', Prog. Theor. Phys. 106 (2001) 561 arXiv:hep-ph/0104258.

[27] D. G. Cerdeño, S. Khalil and C. Muñoz, 'Supersymmetric dark matter and neutralino-nucleon cross section', Proceedings of CICHEP Conference, Cairo (2001), Rinton Press (2001) 214 arXiv:hep-ph/0105180.

[28] R. Arnowitt and B. Dutta, 'Dark matter detection rates in SUGRA models', arXiv:hep-ph/0112157.

[29] D. G. Cerdeño, E. Gabrielli and C. Muñoz, 'Experimental constraints on the neutralino-nucleon cross section', arXiv:hep-ph/0204271.

[30] R. Arnowitt and B. Dutta, 'Susy dark matter: closing the parameter space', arXiv:hep-ph/0210339.

[31] R. Dermisek, S. Raby, L. Roszkowski, R. Ruiz de Austri, 'Dark matter and $B_{s} \rightarrow$ $\mu^{+} \mu^{-}$with minimal $S O_{10}$ soft SUSY breaking', J. High Energy Phys. 04 (2003) 037 arXiv:hep-ph/0304101.

[32] S. Profumo, 'Neutralino dark matter, b-tau Yukawa unification and non-universal sfermion masses' Phys. Rev. D 68 (2003) 015006 arXiv:hep-ph/0304071; 'Neutralino relic density in supersymmetric GUTs with no-scale boundary conditions above the unification scale', J. High Energy Phys. 06 (2003) 052 arXiv:hep-ph/0306119.

[33] H. Baer, C. Balázs, A. Belyaev and J. O'Farril, 'Direct detection of dark matter in supersymmetric models', JCAP 09 (2003) 007 arXiv:hep-ph/0305191; 
H. Baer, A. Mustafayev, S. Profumo, A. Belyaev and X. Tata, 'Direct, Indirect and Collider Detection of Neutralino Dark Matter In SUSY Models with Non-universal Higgs Masses', arXiv:hep-ph/0504001.

[34] A. Corsetti and P. Nath, 'Gaugino mass nonuniversality and dark matter in sugra, strings and D-brane models', Phys. Rev. D 64 (2001) 125010 arXiv:hep-ph/0003186.

[35] V. Bertin, E. Nezri and J. Orloff, 'Neutralino dark matter beyond CMSSM universality', J. High Energy Phys. 02 (2003) 046 arXiv:hep-ph/0210034.

[36] A. Birkedal-Hansen and B. D. Nelson, 'Relic neutralino densities and detection rates with nonuniversal gaugino masses', Phys. Rev. D 67 (2003) 095006 arXiv:hep-ph/0211071;

A. Birkedal-Hansen, 'rSUGRA: putting nonuniversal gaugino masses on the (W)map', arXiv:hep-ph/0306144.

[37] U. Chattopadhyay and D. P. Roy, 'Higgsino dark matter in a SUGRA model with nonuniversal gaugino masses', Phys. Rev. D 68 (2003) 033010 arXiv:hep-ph/0304108.

[38] C. Pallis, ' $b-\tau$ unification and sfermion mass non-universality', Nucl. Phys. B 678 (2004) 398 arXiv:hep-ph/0304047.

[39] D. G. Cerdeño and C. Muñoz, 'Neutralino dark matter in supergravity theories with non-universal scalar and gaugino masses', J. High Energy Phys. 10 (2004) 015 arXiv:hep-ph/0405057.

[40] S. Chen et al. [CLEO Collaboration], 'Branching fraction and photon energy spectrum for $b \rightarrow s \gamma^{\prime}$, Phys. Rev. Lett. 87 (2001) 251807 arXiv:hep-ex/0108032.

[41] H. Tajima [BELlE Collaboration], 'Belle B physics results', Int. J. Mod. Phys. A17 (2002) 2967 arXiv:hep-ex/0111037.

[42] G. Bélanger, F. Boudjema, A. Pukhov and A. Semenov, 'micrOMEGAs: A program for calculating the relic density in the MSSM', Comput. Phys. Commun. 149 (2002) 103 arXiv:hep-ph/0112278; 'micrOMEGAs: Recent developments', arXiv:hep-ph/0210327;

[43] G. W. Bennett et al. [Muon g-2 Collaboration], 'Measurement of the negative muon anomalous magnetic moment to 0.7-ppm', Phys. Rev. Lett. 92 (2004) 161802 arXiv:hep-ex/0401008.

[44] M. Davier, S. Eidelman, A. Höcker and Z. Zhang, 'Updated estimate of the muon magnetic moment using revised results from $e^{+} e^{-}$annihilation', Eur. Phys. J. C 31 (2003) 503 arXiv:hep-ph/0308213; 
[45] K. Hagiwara, A. D. Martin, D. Nomura and T. Teubner, 'Predictions for g-2 of the muon and $\alpha_{Q E D}\left(M_{Z}^{2}\right)^{\prime}$, Phys. Rev. D 69 (2004) 093003 arXiv:hep-ph/0312250.

[46] J. F. de Trocóniz and F. J. Ynduráin, 'The hadronic contributions to the anomalous magnetic moment of the muon', Phys. Rev. D 71 (2005) 073008 arXiv:hep-ph/0402285.

[47] J. Foster, K. Okumura and L. Roszkowski, 'New Higgs effects in B-physics in Supersymmetry with general flavour mixing', Phys. Lett. B 609 (2005) 102 arXiv:hep-ph/0410323. 